\title{
MATÉRIAUX DURS POUR AIMANTS PERMANENTS
}

\author{
H. LEMAIRE \\ Société d'Etudes et de Recherches Magnétiques «SERMAG » \\ Laboratoire de Recherche d'Aimants Ugimag, \\ 1 à 3, rue Alfred-Gueymard, Boîte Postale No 26, \\ 38401 Saint-Martin-d'Hères, France
}

\begin{abstract}
Résumé. - Souvent ignorés, parce que rarement visibles, les aimants permanents sont maintenant devenus des composants indispensables de l'électronique et de l'électrotechnique, que ce soit pour l'emploi grand public (haut-parleurs, fermetures de portes, moteurs à puissance fractionnaire) ou pour le professionnel (appareils de mesure, séparateurs, circuits pour hyperfréquences). Et il faut compter par centaines de millions d'unités les aimants qu'une grande usine est capable de produire chaque année, depuis l'aimant de $4 \mathrm{mg}$ en nuance performante à base de cobalt-terres rares et destiné aux transducteurs de l'horlogerie, jusqu'à l'aimant de $40 \mathrm{~kg}$, coulé en une seule pièce dans la nuance Ticonal 600 , pièce maîtresse du stator d'un générateur. Ce facteur de $10^{7}$ dans le poids se retrouve presque dans les coercivités intrinsèques des ferromagnétiques industriels qui s'étagent de $10^{-2}$ Oe pour les très bons matériaux doux à $10^{5}$ Oe pour les matériaux durs les plus récents. A l'occasion d'une revue des principaux matériaux pour aimants permanents fabriqués couramment, les divers mécanismes concourant à la coercivité intrinsèque des aimants modernes sont présentés et illustrés par des exemples tirés de nos plus récents résultats. Si l'ancienne théorie de l'accrochage des parois de Bloch, développée autrefois pour les aciers, revient à la mode avec les alliages cobalt-terres rares substitués au cuivre et au fer, l'origine des propriétés de la plupart des aimants modernes s'interprète en termes de théorie des fines particules : barrière au renversement de l'aimantation, gouvernée par l'anisotropie de forme (Ticonals, Aimants en poudre) ou l'anisotropie monocristalline (Platine-Cobalt, Ferrites dures) - barrière à la nucléation de domaines inverses ou au décrochage de parois (Aimants Cobalt-Terres Rares frittés). L'examen des courbes de première aimantation, des courbes de désaimantation intrinsèque, des cycles de recul, permet de différencier ces mécanismes de coercivité, que l'on illustre par la présentation de résultats relatifs aux nuances à forte coercivité comme le nouveau Ticonal 2000 à grande énergie spécifique $\left((B H)_{\max }=6,5 \mathrm{MG} . \mathrm{Oe}\right)$, le Platine-Cobalt Sermalloy $\mathrm{P}$, le caoutchouc magnétique totalement orienté $\left((B H)_{\max }=2 \mathrm{MG}\right.$.Oe) et surtout les aimants Cobalt-Terres Rares Coramag. Alors que, jusqu'à ces dernières années, l'augmentation de coercivité s'accompagnait toujours d'une baisse de la rémanence, l'apparition des composés intermétalliques de cobalt et de terres rares renverse cette situation et permet d'atteindre facilement des énergies spécifiques très élevées. La préparation, les possibilités de mise en forme mécanique et magnétique, les propriétés thermiques des aimants cobalt terres rares sont présentées et discutées.
\end{abstract}

Abstract. - Often ignored, for unseen, permanent magnets are increasingly becoming valuable components for electronical and electrotechnical devices, used in a large scale for loud-speakers, doors latches, low power motors, ..., as well as in measuring systems, separators, microwaves devices. The total number of unit magnets produced per year in a large plant amounts to several hundreds of millions, since the small magnet weighing $4 \mathrm{mg}$ in a high quality material as CobaltRare Earth and used in electric watches, till the big $40 \mathrm{~kg}$ magnet, directly cast in Ticonal 600 , for the stator of an electric generator. The same $10^{7}$ factor in the weight can be found in the coercivities of ferromagnetic materials, going from $10^{-2}$ Oe for very soft materials to $10^{5}$ Oe for very hard materials. This review deals with the various hard materials now in production, illustrated by a few new laboratory results. If the old theory of pinned Bloch walls, initially developed for magnet steels, comes back again for cobalt-rare earths alloys substituted with copper and iron, the origin of the properties of most modern hard materials is interpreted in terms of single domain fine particle theory : in one case, magnetization reversal is governed, either by a shape anisotropy (Ticonals, powder magnets) or a crystal anisotropy (platinum-cobalt alloy, hard ferrites) - in the other case, it is governed by nucleation of reversed domains or unpinning of Bloch walls (sintered cobalt-rare earths magnets). The study of first magnetization curves, as well as intrinsic curves or recoil curves, is very useful to distinguish between various mechanisms of coercivity, and is illustrated here by new results obtained on high coercivity materials, as Ticonal 2000 $\left(\left(B H_{\max }\right)=6.5 \times 10^{6} \mathrm{G}\right.$.Oe $)$, Sermalloy P, Plasto-Ferrite magnets Ferriflex 10 very well oriented $\left((B H)_{\max }=2.0 \times 10^{6} \mathrm{G} . \mathrm{Oe}\right)$ and Cobalt-Rare Earth Coramag. Although an increase in coercivity is usually followed by a decrease in remanence, this does not appear in intermetallic compounds based on transition and rare earth metals, thus allowing very high specific energies. Synthesis, mechanical and magnetic shaping, thermal properties of these compounds are showed and discussed. 
1. Introduction. - Pour un nouvel observateur, le monde de l'industrie des aimants apparaît à la fois étrangement conservateur et progressiste, car, que ce soit dans la nature des matériaux ou dans la forme des aimants (Fig. 1), la tradition y est solidement

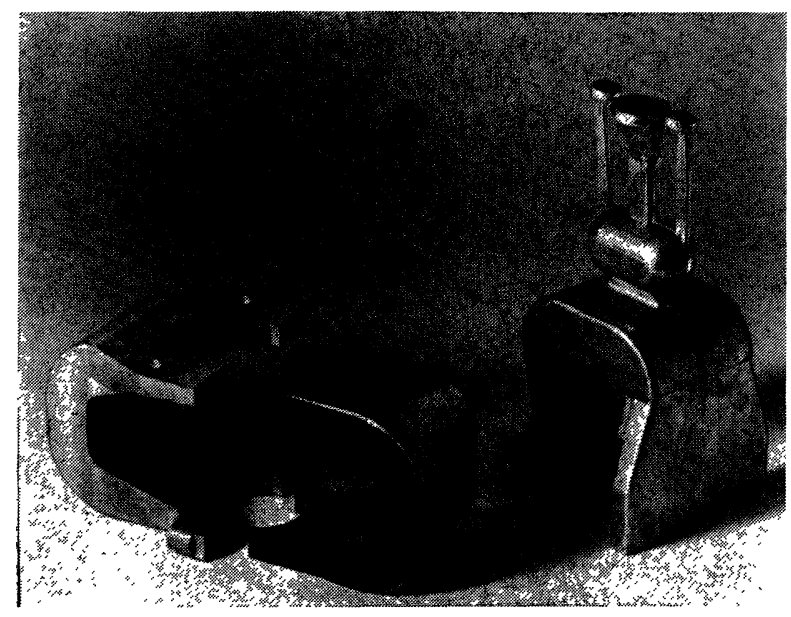

Fig. 1. - Aimant traditionnel en $\mathbf{U}$, pour magnétron, en Ticonal 600 (au centre), après enrobage en alliage léger (à droite), complètement usiné (à gauche).

ancrée. Il n'est pas d'exemple de matériaux qui, ayant atteint le stade industriel, ne soient plus à l'heure actuelle en production, tout au moins dans une partie du monde. Les aciers martensitiques des sièclès passés conservent encore la faveur des fabricants de moteurs à hystérésis, de par leur faible coercivité et leur forte perméabilité, et ils contribuent malheureusement à maintenir dans l'esprit du public, même très éduqué, l'image désastreuse de l'aimant non permanent. Les forces de l'habitude ou de l'inertie, l'adéquation des structures industrielles, font encore du Remalloy (Fe-Co-Mo) et du Cu-Ni-Fe, les composants de base des téléphones et tachymètres de voiture américains. L'avènement des Cobalt-Terres Rares n'a pas tué le coûteux Platine-Cobalt, dont la garantie de stabilité assure la pérennité d'une faible production (250 kg/an) pour les tubes électroniques de l'espace. Ce conservatisme, latent à toute entreprise humaine, n'est pas sans avantages pour les producteurs : la substitution de l'excitation magnéto-statique à l'excitation électro-dynamique a amené le développement énorme des ferrites dures pour petits moteurs, lequel trouve son prolongement naturel dans les moteurs de puissance, où le courant continu tient la dragée haute au courant alternatif [1].

Progressiste, le monde des aimants l'est aussi, car il s'est écoulé moins de dix ans entre la première observation d'une coercivité intrinsèque énorme dans les poudres cobalt-terres rares [2], [3] et la production industrielle d'aimants permanents basés sur elle [4] ; peu d'idées nouvelles ont eu une adolescence aussi courte. Sur le plan même des concepts théoriques, l'évolution se fait sentir, qui substitue aux paramètres électrotechniques de l'induction B et de la coercivité d'induction ${ }_{B} H_{\mathrm{c}}$, les paramètres beaucoup plus physiques de l'aimantation $\mathbf{M}$ et de la coercivité d'aimantation ${ }_{i} H_{\mathrm{c}}$. En même temps se développent de nouveaux modèles de la coercivité et s'amorce un virage historique (Fig. 2) qui laisse entrevoir ce que seront les aimants de l'avenir. Alors que la croissance de la coercivité s'était jusqu'à maintenant accompagnée d'une baisse des aimantations

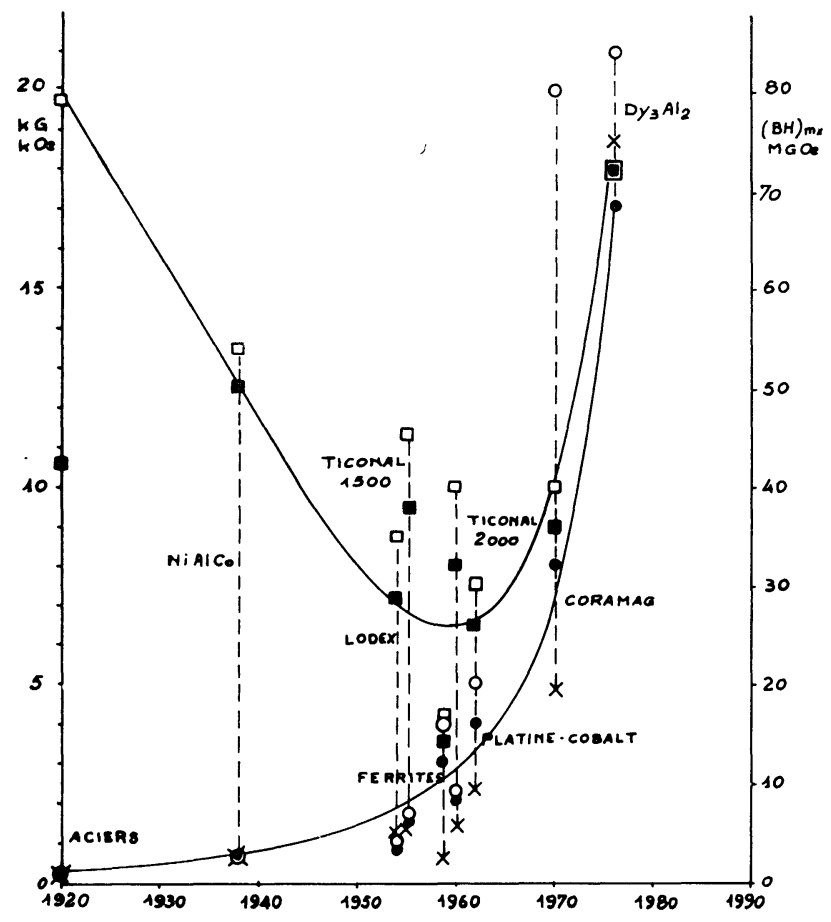

FIG. 2. - Evolution des propriétés des aimants permanents : la coercivité intrinsèque se démarque de plus en plus de la coercivité d'induction, les aimantations à saturation et rémanente augmentent. $\square$ Aimantation à saturation; Aimantation rémanente ; Coercivité d'induction; $\bigcirc$ Coercivité intrinsèque ; $\times$ Energie spécifique maximum.

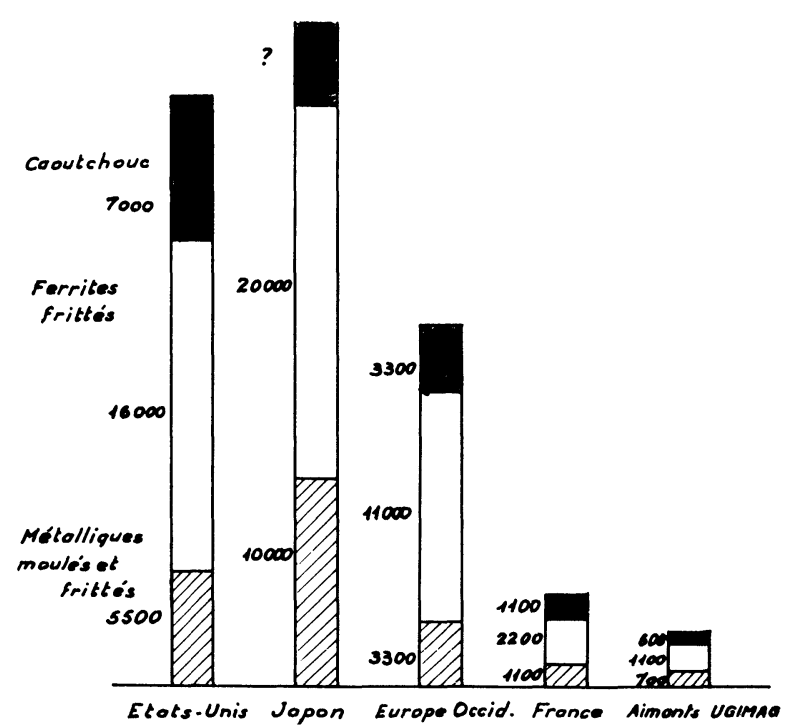

Fig. 3. - Répartition en tonnage de la production mondiale d'aimants en 1972. 


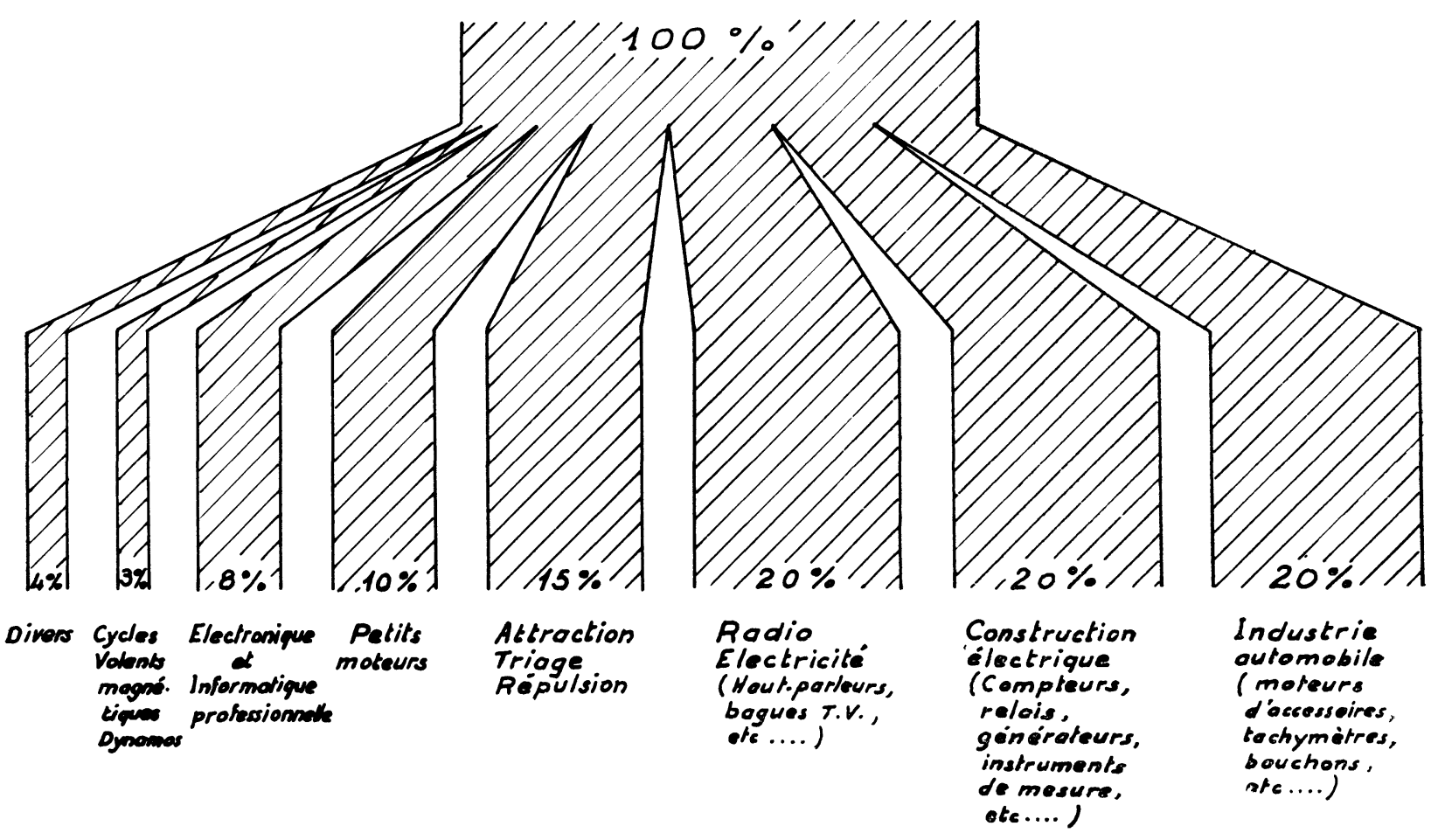

FIG. 4. - Répartition par utilisations de la production d'aimants. Les oxydes durs pour bandes magnétiques ne sont pas inclus.

à saturation et rémanente dans la succession aciersTiconals-Ferrites dures-Platine-Cobalt, la tendance vient de se renverser avec les aimants à base de métaux de transition et de terres rares : la rémanence et l'aimantation à saturation augmentent de nouveau. Le record actuel est détenu par $\mathrm{Dy}_{3} \mathrm{Al}_{2}$ qui offre, à la température de $4,2 \mathrm{~K}$, la performance record d'une énergie spécifique de $72 \times 10^{6}$ G.Oe [5], tant attendue autrefois [6] pour les Ticonals. Il est certain que ces valeurs extraordinaires seront atteintes dans un avenir proche par des matériaux capables de travailler à la température ambiante, comme $\operatorname{Pr}_{2}\left(\mathrm{Co}_{0,7}-\mathrm{Fe}_{0,3}\right)_{17}$ [7], une fois que seront compris et maîtrisés les mécanismes de nucléation de domaines inverses $\left(B_{\mathrm{s}}=16400 \mathrm{G}\right.$, $\left.(B H)_{\max }=67 \times 10^{6} \mathrm{G} . \mathrm{Oe}\right)$.

Les figures 3 et 4 permettent de se rendre compte de la production mondiale d'aimants permanents et de leur destination. En tonnage, les chiffres sont modestes, comparés à celui de la production d'acier brut, mais la valeur ajoutée est considérable, compte tenu de la faible taille unitaire et de la complexité des processus de fabrication. Le poids moyen de l'aimant permanent est très faible, de l'ordre d'une dizaine de grammes; on trouve aussi bien le petit aimant de $4 \mathrm{mg}$ destiné au rotor d'une montre électronique que le gros aimant de $40 \mathrm{~kg}$, coulé en une seule pièce dans la nuance Ticonal 600 et qui équipe le stator d'un générateur. Les principaux matériaux se classent en trois grandes catégories : - les aimants métalliques, dont le leader Ticonal, après avoir connu un fléchissement au milieu des années 60 , a atteint maintenant un palier et s'y maintient, grâce à son excellente stabilité thermomagnétique et à son aptitude à se conformer aux petites séries - les aimants frittés, essentiellement des ferrites hexagonales de baryum et de strontium, qui connaissent un développement prodigieux dans les petits moteurs de grande série et les dispositifs d'attraction - les aimants souples, à base de poudres de ferrites et de caoutchouc, qui, longtemps confinés à la seule fermeture de frigidaire où la grande surface compense la faiblesse des propriétés magnétiques, effectuent maintenant une percée dans les autres domaines, grâce à une amélioration sensible de leurs caractéristiques. La figure 4 est un peu trompeuse, qui n'accorde que $8 \%$ au marché des aimants en électronique et informatique professionnelle : il faut $y$ entendre, soit des aimants de champ soit des aimants de moteurs, rotatifs ou linéaires. Dans cette répartition n'est pas pris en compte le tonnage considérable d'oxyde ferrimagnétique $\gamma-\mathrm{Fe}_{2} \mathrm{O}_{3}$, consommé dans les bandes magnétiques d'enregistrement et qui appartient, par ses caractéristiques magnétiques (une coercivité de 100 à $600 \mathrm{Oe}$ ) au domaine des aimants permanents : le chiffre d'affaires mondial est de quatre fois celui de l'ensemble des matériaux durs [8] en augmentation constante avec l'accroissement des besoins de stockage et de diffusion des informations créé par l'essor de l'informatique.

2. Les mécanismes de la coercivité et le renversement de l'aimantation. - Sans entrer dans les détails [9], il paraît utile de rappeler brièvement les prin- 
cipaux mécanismes de la coercivité ${ }_{i} H_{\mathrm{c}}$ des aimants permanents, ou grandeur du champ démagnétisant nécessaire à l'annulation de l'aimantation macroscopique $\mathbf{M}$ d'un échantillon du matériau considéré, après passage à la saturation par application d'un champ en principe infini. Cette définition n'est d'ailleurs pas sans ambiguïté en faisant intervenir la taille de l'échantillon, surtout dans un domaine où l'ordre de grandeur de l'énergie magnétostatique d'un volume donné; comparée aux énergies cristalline, de forme, de tension, est essentiel dans la détermination des processus de croissance, de stabilisation et de décroissance de l'aimantation: la coercivité du fer peut passer de 10000 Oe à 0,01 Oe selon que l'on s'adresse à un petit ou à un grand monocristal.

Une notion fondamentale à la compréhension des matériaux pour aimants permanents est le concept de domaines magnétiques. Les domaines magnétiques sont des régions du matériau, où les moments magnétiques sont tous orientés dans la même direction, y compris en l'absence de champ directeur externe. Entre deux domaines magnétiques adjacents prend place une zone de transition dans laquelle la direction d'alignement des moments atomiques d'un domaine magnétique passe à celle du domaine magnétique voisin, soit par une altération progressive de l'orien- tation sur une zone appelée paroi de Bloch, soit par un changement brutal de l'orientation (séparation spatiale avec disparition locale de l'aimantation, joints de grains à structure cristalline perturbée, paroi étroite). Dans un cas comme dans l'autre, pour atteindre l'état coercitif, il faudra passer, soit par la nucléation de domaines magnétiques inverses et leur extension progressive par déplacements de parois, soit par le retournement, brutal ou progressif, d'une moitié du volume aimanté. Ces différents mécanismes vont se distinguer entre eux par les apparences différentes qu'ils donnent aux courbes de première aimantation, aux courbes de désaimantation et aux courbes de recul, ces dernières étant obtenues par décroissance du champ démagnétisant dans le deuxième quadrant.

La figure $5 a$ illustre le principe de la coercivité par accrochage de parois: dans un cristal, deux domaines magnétiques inverses sont séparés par une paroi de Bloch, dont l'énergie, compromis entre les énergies d'échange et d'anisotropie, dépend de la position dans le cristal. D'abord accrochée en A, la paroi se déplacera brutalement en $B$, lorsque la pression exercée par le champ sera supérieure à la force de rappel créée par le défaut, source du minimum d'énergie proche de A. L'aimantation sera d'autant $a$

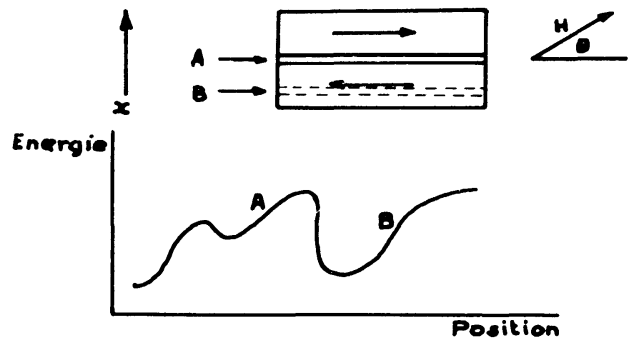

$c$

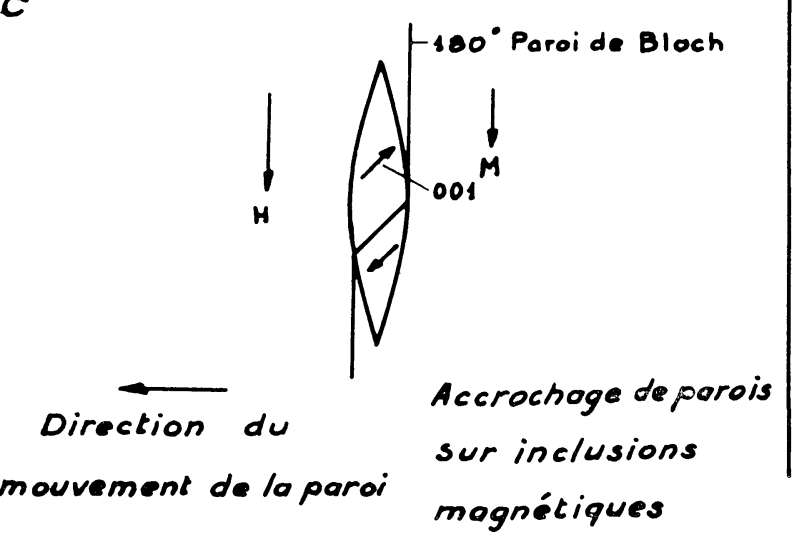

b

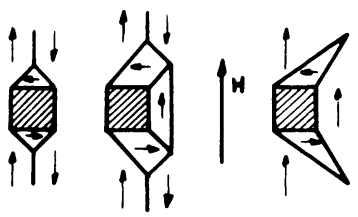

Accrochage de parois sur

inclusions non magnétiques

$d$

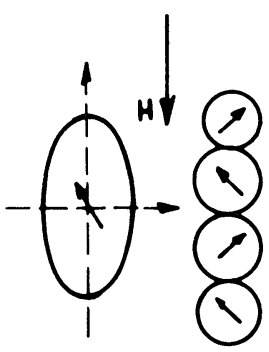

Rototion

cohérente
Chaine de sphicres

"Fonning"

FIG. 5. - Les mécanismes classiques de la coercivité. $a$ ) L'état d'énergie minimum dépend de la position de la paroi. $b$ ) Accrochage sur inclusion non magnétique, avec domaines de fermeture minimisant l'énergie magnéto-statique. c) Accrochage sur inclusion magnétique à forte anisotropie. $d$ ) Rotations cohérente et par «fanning ». 
plus lente que la densité spatiale des défauts sera grande, l'aimantation et la désaimantation seront d'autant plus difficiles que la force de rappel sera grande. La figure $5 b$ illustre le mécanisme de coercivité par inclusions non magnétiques où l'abaissement d'énergie et l'augmentation de la force de rappel se font simultanément par diminution de la surface de la paroi [10] et diminution de l'énergie magnétostatique [11] avec création de domaines magnétiques de fermeture du flux [12]. Dans la figure $5 c$ est représenté le mécanisme supplémentaire proposé [13] pour expliquer la coercivité du Platine-Cobalt. Dans un échantillon massif de structure cubique à faces centrées sont dispersés au hasard des domaines cristallins à structure tétragonale ordonnée en cohérence avec la matrice ; les deux phases sont magnétiques, mais d'aimantations et d'anisotropies différentes, et les parois en déplacement s'accrochent sur ces domaines cristallins où règne la plus forte anisotropie cristalline $\left(2 \times 10^{7} \mathrm{erg} / \mathrm{cm}^{3}\right.$ contre $\left.6 \times 10^{5} \mathrm{erg} / \mathrm{cm}^{3}\right)$. La figure 6 est la classique courbe d'hystérésis, obtenue pour un échantillon de Sermalloy $P$ [14] à base de platine-cobalt, et qui illustre bien les processus d'aimantation et de désaimantation par déplacement et accrochage de parois. L'aimantation de la matrice, essentiellement composée de la phase cubique à faces centrées et à faible anisotropie, ne se fait que lentement par déformation de parois, celles-ci restant accrochées sur les petits domaines cristallins à structure tétragonale et forte anisotropie. Il faut atteindre un champ d'au moins 4700 Oe pour voir croître rapidement l'aimantation et apparaître la coercivité, ce champ étant nécessaire pour décrocher la paroi ancrée sur une direction (001) de la phase tétragonale [13]. La fin de l'aimantation se traduit par la rotation des moments atomiques vers la direction préférentielle imposée par le champ. Au retour, le rapport

$$
B_{\mathrm{r}}(H=0) / B_{\mathrm{s}}(H=30000)=0,87
$$

reste comparable à celui attendu pour une matrice cubique à faces centrées de constante d'anisotropie négative, les moments relaxant vers les quatre directions (111). Joint à la symétrie complète de la courbe d'hystérésis, à son indépendance avec le mode de désaimantation appliqué, thermique ou magnétique oscillant, ou avec l'application préalable d'un pulse d'aimantation de $80 \mathrm{kOe}$, ce résultat démontre que la phase tétragonale uniaxe $\left(B_{\mathrm{r}} / B_{\mathrm{s}}=0,5\right)$ ne contribue que peu à l'aimantation, tout en étant fondamentale pour la coercivité. Un modèle basé sur les fines particules monodomaines [15] ne s'applique donc pas. Quant aux courbes de recul, elles traduisent une légère réaimantation en champ négatif décroissant, provoquée par un relâchement de la tension appliquée aux parois et un retour des moments atomiques vers leurs axes préférentiels.

A la limite, lorsque l'augmentation des énergies d'échange et d'anisotropie ne peut plus être compensée par une diminution supérieure de l'énergie magnéto- statique, le monocristal devient monodomaine. Ceci se produit pour une taille et une forme, fonctions des ordres de grandeur des énergies mises en jeu et cet effet de taille n'est pas sans rappeler un résultat de mécanique quantique, à savoir l'allure des fonctions d'onde d'une particule dans un puits de potentiel à un seul minimum (puits carré, oscillateur harmonique..., etc.). Pour une énergie donnée, l'allure de la fonction d'onde n'a qu'un seul maximum si le puits est de faible dimension (mouvement de point zéro), tout comme la particule monodomaine n'a qu'une seule direction d'aimantation; en augmentant la largeur du puits, à la même énergie, correspond maintenant une fonction à plusieurs maxima, successivement positifs et négatifs, de même qu'un cristal de grandes dimensions se scinde en une structure polydomaine d'aimantations alternées. Cette analogie, pas encore signalée à notre connaissance, mérite certainement d'être approfondie et permettrait peutêtre de résoudre le problème de l'état d'équilibre d'un cristal ferromagnétique, par une approche entièrement nouvelle. En poussant le raisonnement, on peut même rechercher l'équivalent quantique du grain superparamagnétique, qui apparaît lorsque la taille du monodomaine est devenue suffisamment faible pour que l'énergie thermique contrebalance l'énergie d'anisotropie.

Dans le grain monodomaine, l'énergie d'anisotropie peut avoir pour origine la forme ou l'interaction aimantation-cristal, d'origine intrinsèque ou de tension. La première est schématisée sur la figure $5 d$ : pour un ellipsoïde allongé, l'énergie magnétostatique est plus faible selon le grand axe que selon un diamètre, et l'aimantation s'y alignera préférentiellement. Pour la retourner, il faudra franchir une barrière d'énergie ; dans un matériau macroscopique, ceci se traduit par l'apparition [16] d'une coercivité proportionnelle à l'aimantation qui peut atteindre la valeur élevée de 10700 Oe dans le cas du fer, si la rotation se fait de manière cohérente (tous les moments atomiques restent parallèles). Une agglomération de telles particules est à la base des aimants en poudres agglo-

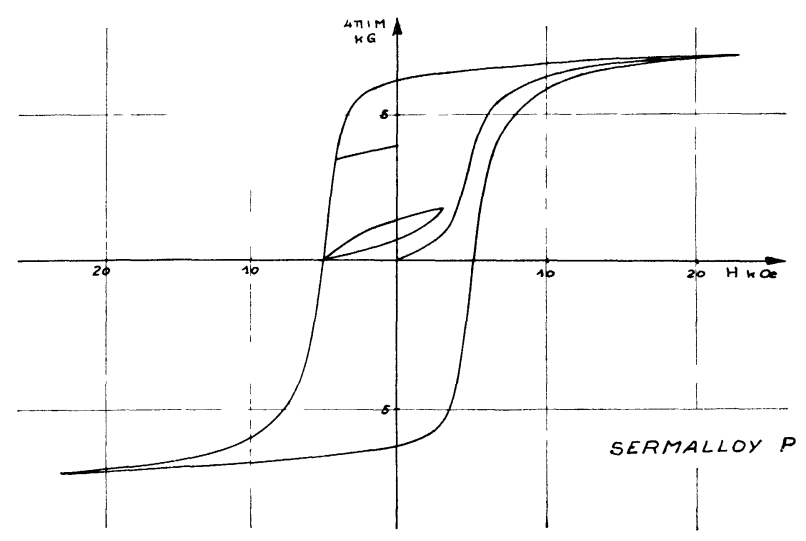

FIG. 6. - Courbes classiques de première aimantation et d'hystérésis, mesurées sur un échantillon de Platine-Cobalt. Noter la symétrie de la courbe d'hystérésis. 
mérées de fer et fer-cobalt [17], des bandes magnétiques à base de $\gamma-\mathrm{Fe}_{2} \mathrm{O}_{3}$ et des aimants Ticonal où la structure en particules est obtenue par une décomposition spinodale en phase solide. Mais la coercivité est loin d'atteindre la valeur donnée plus haut, pour plusieurs raisons. En premier, la forme des particules est loin d'être celle de l'ellipsoïde parfait. Elle se rapproche très souvent de la sphère à anisotropie nulle, ou de la chaîne de sphères visualisée sur la

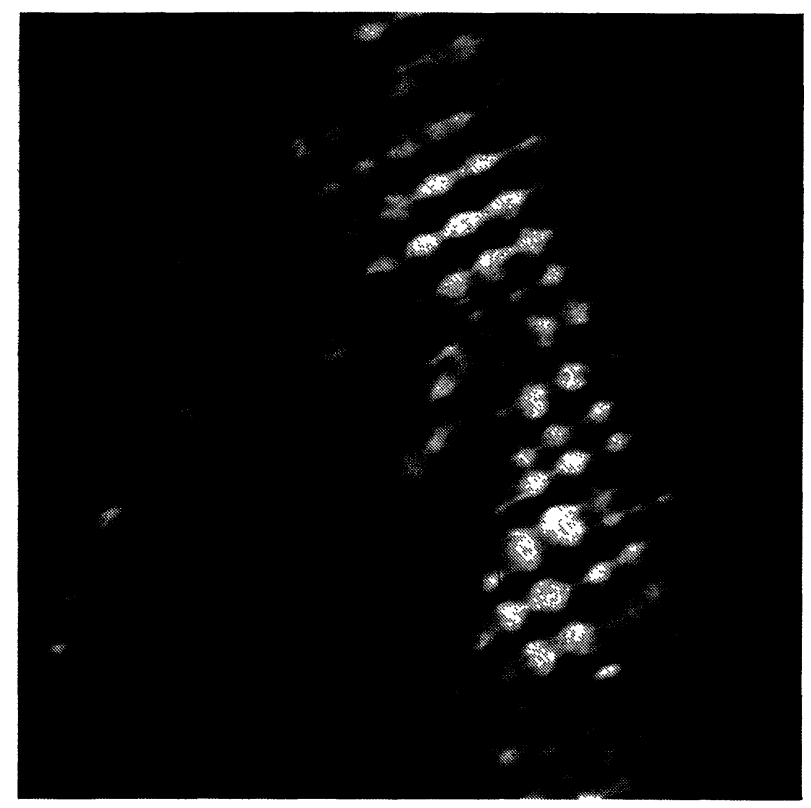

(a)

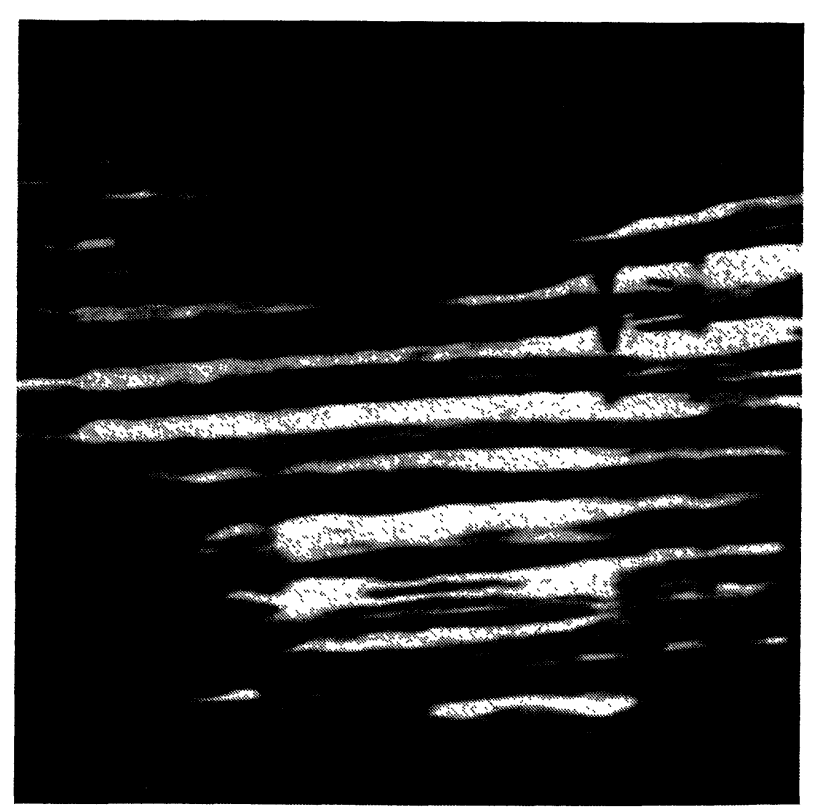

(b)

Fig. 7. - Examens au microscope électronique de lames minces de Ticonal 2000. En haut, après traitement magnétique isotherme, les chaînes de sphères responsables d'une coercivité par «fanning » de 1000 Oe sont bien visibles. En bas, après revenu, le développement d'ellipsoïdes allongés a fait croître la coercivité à $2000 \mathrm{Oe}$. figure $5 d$. Par interaction magnétique dipolaire d'une sphère sur l'autre est alors favorisé un mécanisme de retournement non cohérent ou «fanning», qui abaisse la coercivité à un chiffre compris entre le dixième et le quart de la valeur de l'ellipsoïde de même dimension [18]. Ce fait est très bien illustré par le haut de la figure 7, où les chaînes de sphères sont observées [19] au microscope électronique sur une lame mince de Ticonal 2000 [14], juste après le traitement thermo-magnétique isotherme : dans cet état, la coercivité de l'alliage n'atteint que 1000-1 200 Oe. En second lieu, le renversement cohérent fait apparaître de l'énergie magnétostatique en développant des pôles sur la surface de la particule; pour des tailles assez grandes, un autre mécanisme, le «curling $"$, prend place [20], où les moments magnétiques tournent en restant parallèles à la surface de l'échantillon. Ce mécanisme conduit à des coercivités inférieures à celle du «fanning » lorsque la taille croît.

Une autre anisotropie a pour origine l'interaction mono-cristalline, dont on parlera un peu plus en détail pour les ferrites et les aimants cobalt-terres rares. Dans le tableau I sont résumés les principales sources de la coercivité et un classement de quelques matériaux importants selon la contribution dominante à la coercivité. Certains matériaux, comme le Vicalloy (Fe-Co-V), n'ont pas encore pu être classés, tant les différentes contributions donnent des ordres de grandeur comparables. D'autres trouvent leur coercivité dans la cohabitation simultanée de deux mécanismes, comme le Platine-Cobalt dont nous venons de parler, où l'accrochage de parois se fait sur des inclusions à forte anisotropie cristalline. Un autre cas très intéressant est celui des aimants cobalt-terres rares qui, selon leur composition, se placent dans deux familles tout à fait différentes. La figure 8 compare les courbes

TABLEAU I

Les principales sources de coercivité

$$
\begin{array}{lr}
\text { - Contraintes ou inclusions }{ }_{J} H_{\mathrm{c}} \propto \frac{1}{J_{\mathrm{s}}} \\
\text { - Forme } & { }_{J} H_{\mathrm{c}} \propto J_{\mathrm{s}} \\
\text { - Magnéto-cristalline } & { }_{\mathrm{J}} H_{\mathrm{c}} \propto \frac{K}{J_{\mathrm{s}}}
\end{array}
$$

\section{Contribution dominante au champ coercitif}

\begin{tabular}{lccc}
\multicolumn{1}{c}{ Aimant } & $\begin{array}{c}\text { Contraintes } \\
\text { (inclusions) }\end{array}$ & Forme & $\begin{array}{c}\text { Magnéto- } \\
\text { cristalline }\end{array}$ \\
Aciers & $\bar{X}$ & - & - \\
Cunife & & $\mathrm{X}$ & \\
Ticonal & & $\mathrm{X}$ & \\
$\begin{array}{l}\text { Poudres de Fe, Fe-Co } \\
\text { Platine-Cobalt }\end{array}$ & $\mathrm{X}$ & $\mathrm{X}$ & $\mathrm{X}$ \\
$\begin{array}{l}\text { Ferrites } \\
\text { Cobalt-Terres Rares }\end{array}$ & & & $\mathrm{X}$ \\
$\quad\left(\mathrm{SmCO}_{5}\right)$ & & & $\mathrm{X}$ \\
Cobalt-Terres Rares & $\mathrm{X}$ & & $\mathrm{X}$
\end{tabular}



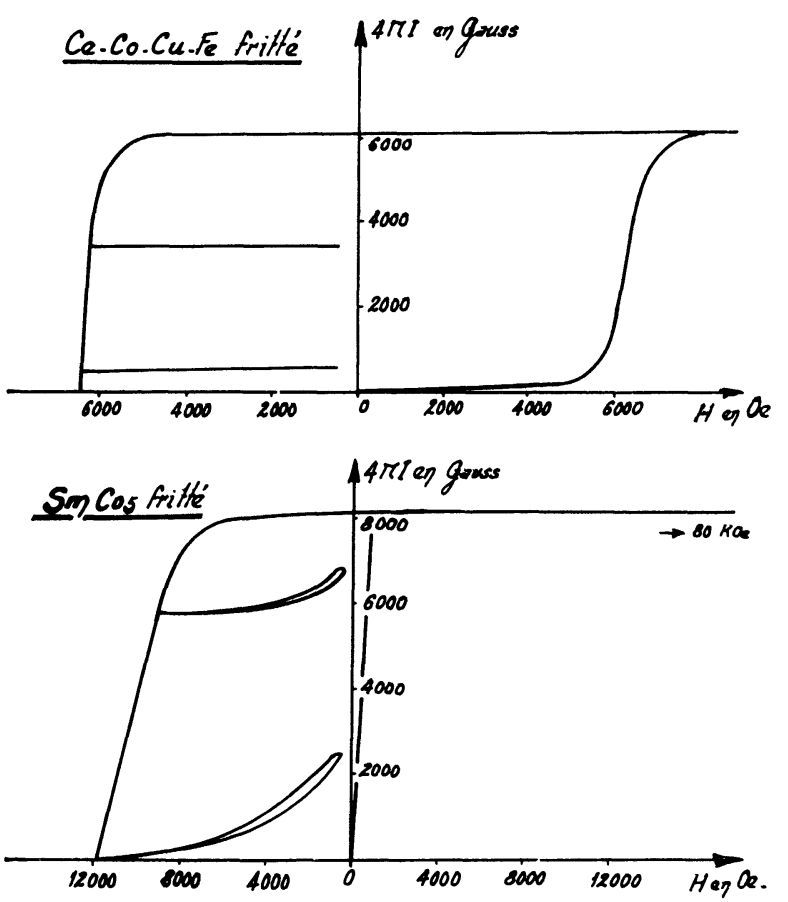

Fig. 8. - Courbes d'aimantation, de désaimantation et de recul d'aimants cobalt-terres, traduisant la différence des origines de la coercivité.

de première aimantation, de désaimantation et de recul de ces aimants, obtenus soit par la voie métallurgique (terre rare-cobalt-cuivre-fer) soit par la voie des poudres (terre rare-cobalt fritté). Les comportements sont complètement différents. Dans le premier cas, où l'on étudie un monocristal, l'aimantation reste très faible, pour ne croître brutalement que lorsqu'un champ seuil d'environ 6000 Oe est atteint. Au retour, l'aimantation reste constante et ne s'inverse brusquement que pour un champ égal au champ seuil ; la coercivité obtenue est indépendante du champ de saturation appliqué. Tout ceci s'explique très bien par un accrochage de parois dans un milieu magnétique fortement anisotrope sur une dispersion très fine de particules d'aimantation différente et d'anisotropie faible [21, 22], plus précisément de particules à structure $\mathrm{TR}_{2} \mathrm{M}_{17}$ dans une matrice $\mathrm{TRM}_{5}$. C'est le vieux mécanisme de durcissement des aciers d'autrefois, avec toutefois deux variantes : compte tenu de la grande anisotropie des structures $\mathrm{CaCu}_{5}$, la réduction de l'énergie de paroi par diminution de surface est à elle seule suffisante pour expliquer la coercivité sans faire appel à une réduction de l'énergie magnétostatique - la distribution des particules dans la matrice est régulière, ce qui assure une unicité du champ coercitif et explique dans le monocristal la parfaite rectitude des courbes de recul, à l'opposé de ce qui est constaté pour le Platine-Cobalt (Fig. 6). $\mathrm{Au}$ contraire, dans le cas du samarium-cobalt fritté, la saturation est atteinte immédiatement, dans un champ très faible auquel ne s'oppose que le champ démagnétisant du grain. Cependant, la coercivité importante n'est atteinte que si le champ appliqué est suffisant pour chasser la paroi hors du grain ou l'accrocher solidement sur des défauts de surface ou de joints [23, 24]; lors du retour en champ nul, ou négatif la très forte anisotropie cristalline de ces composés $\left(H_{\mathrm{A}} \sim 3 \times 10^{5} \mathrm{Oe}\right)$ s'oppose aux retournements locaux d'aimantation, réalisant ainsi le "paradoxe de Brown" [25] d'une coercivité indépendante de la taille du grain pour les systèmes à forte anisotropie. Quant aux courbes de recul, après un départ tangentant l'horizontale et traduisant bien la constance de l'aimantation, elles montrent le phénomène unique d'une hystérésis prononcée, due à la répartition des coercivités [26, 27] des différents grains constituant l'échantillon : les grains de faible coercivité se réaimantent sous l'action des grains non encore retournés.

Nous reviendrons plus loin sur les propriétés des aimants cobalt-terres rares qui constituent à eux seuls une révolution dans le domaine des aimants permanents. Terminons ce chapitre en disant que le paradoxe de Brown est magnifiquement réalisé dans les matériaux à parois étroites comme $\mathrm{Dy}_{3} \mathrm{Al}_{2}$ [5]: l'énergie d'anisotropie $\left(H_{\mathrm{A}} \sim 4,5 \times 10^{5} \mathrm{Oe}\right)$ étant supérieure à l'énergie d'échange, la paroi ne s'étend que sur une seule distance interatomique. A son déplacement s'oppose toute l'énergie d'anisotropie, créant ainsi une barrière très élevée et conduisant à l'aimant le plus puissant jamais réalisé (Fig. 2). Il est possible que, dans un proche avenir, d'autres matériaux soient découverts, où la coercivité serait basée sur ce principe.

3. Le Ticonal 2000. - Avec le tiers de la production mondiale d'aimants (Fig. 3), les aimants métalliques Ticonal se placent parmi les meilleurs matériaux classiques, en ce qui concerne l'induction rémanente (de 8000 à $13000 \mathrm{G}$ ) et le facteur de mérite $(B H)_{\max }$, (de 5 à $12 \times 10^{6} \mathrm{G} . \mathrm{Oe}$ ). Le point de départ fut un alliage $\mathrm{Ni}^{25} \mathrm{Al}^{12} \mathrm{Fe}$ découvert au Japon en 1931 [28]. Avec une coercivité de 400 Oe et un $(B H)_{\max }$ de $1,3 \times 10^{6} \mathrm{G}$. Oe, il surclassait tous ses contemporains ; aussi, très vite, les recherches d'additions bénéfiques ou de traitements thermiques spéciaux se multiplièrent. La découverte essentielle fut l'effet du cobalt. A l'accroissement attendu de l'aimantation à saturation est venu se superposer un accroissement considérable de la rémanence par refroidissement de l'alliage sous champ magnétique [29]. A haute température, l'alliage est homogène en phase cubique centrée. Lors du refroidissement, il se produit une décomposition spinodale en fines particules de fer-cobalt ferromagnétique dans une matrice nickel-aluminium amagnétique, les structures restant cubiques centrées. Si cette démixtion commence en dessous du point de Curie et est faite en présence d'un champ magnétique, ces particules s'allongent considérablement le long de l'axe (100) le plus proche de la direction du champ; tous ces axes pourront d'ailleurs être alignés si l'on 


\section{TABLEAU II}

\begin{tabular}{|c|c|c|c|c|c|}
\hline & $\begin{array}{c}\text { Pas de champ } \\
\text { avec } \\
\text { revenu } \\
-\end{array}$ & $\begin{array}{l}\text { Avec Champ } \\
\text { Sens } \\
\text { d'anisotropie } \\
\end{array}$ & $\begin{array}{l}\text { sans Revenu } \\
\text { Sens } \\
\text { perpendiculaire }\end{array}$ & $\begin{array}{l}\text { Avec Champ } \\
\text { Sens } \\
\text { d'anisotropie }\end{array}$ & $\begin{array}{c}\text { avec Revenu } \\
\text { Sens } \\
\text { perpendiculaire }\end{array}$ \\
\hline $\begin{array}{l}\text { Aimantation à satura- } \\
\text { tion en } G\end{array}$ & 10200 & 10500 & 10500 & 9900 & 9900 \\
\hline $\begin{array}{l}\text { Aimantation réma- } \\
\text { nente }\end{array}$ & 6600 & 8600 & 5100 & 8200 & 4000 \\
\hline $\begin{array}{l}\text { Champ Coercitif in- } \\
\text { trinsèque en Oe }\end{array}$ & 1180 & 1250 & 670 & 1950 & 1400 \\
\hline $\begin{array}{l}\text { Champ coercitif d'in- } \\
\text { duction } \\
\text { Energie snécifique en }\end{array}$ & 1100 & 1100 & 580 & 1840 & 1050 \\
\hline $\begin{array}{l}\text { Energie spécifique en } \\
10^{-6} \mathrm{G} . \mathrm{Oe}\end{array}$ & 2,6 & 2,7 & Non déterminé & 6,3 & Non déterminé \\
\hline Pente à l'origine & 1,9 & 4,1 & 9,6 & 0,8 & 1,8 \\
\hline Point d'inflexion en $\mathrm{Oe}$ & 1350 & 1420 & N'existe pas & 2080 & 2280 \\
\hline Pente à l'inflexion & 6,8 & 11,9 & N'existe pas & 17,1 & 2,7 \\
\hline Pente à la rémanence & 2,1 & 2,2 & 5,7 & 1,0 & 2,2 \\
\hline Pente de recul & 2,4 & 3,8 & 5,6 & 1,0 & 1,9 \\
\hline
\end{tabular}

favorise, lors de la coulée ou ultérieurement par refusion de zone, une croissance cristalline le long de la direction préférentielle, par cristallisaton colonnaire. On se retrouve donc avec un système de particules à anisotropie de forme, dont la coercivité serait maximum pour une taille voisine de celle du monodomaine et dont la rémanence sera exaltée dans la direction du champ. Ces particules sont bien visibles sur la figure 7 , où le revenu a essentiellement pour effet de transformer des chaînes de sphères pontées selon la direction du champ en particules ellipsoïdales allongées : la coercivité croît de 1000 à $2000 \mathrm{Oe}$, en passant du mécanisme de «fanning » à celui de "curling », décrits plus haut. Le tableau II présente quelques chiffres caractéristiques des courbes d'aimantation et de désaimantation de quelques échantillons prélevés dans une coulée à grains cristallins orientés au hasard, et traités dans les conditions suivantes: traitement isotherme sans champ vers

$800^{\circ} \mathrm{C}$ et revenu (échantillon isotrope), traitement isotherme avec champ et sans revenu, traitement isotherme avec champ et revenu (échantillons anisotropes, mesurés parallèlement et perpendiculairement au champ orienteur). Pour l'échantillon isotrope, les courbes obtenues sont assez semblables à celle du Platine-Cobalt (Fig. 6) discutée précédemment, avec un rapport des aimantations rémanente et à saturation compris entre celui du «fanning » pur d'un système cubique (soit 0,832 ) et celui de particules allongées disposées au hasard (soit 0,5 ) ; quant à l'aimantation, elle ne croît nettement qu'à partir d'un champ de 1350 Oe auquel correspond un point d'inflexion sur la courbe de première aimantation (voir aussi Fig. 10). L'application d'un champ pendant le traitement isotherme se fait nettement sentir en différenciant considérablement les deux directions privilégiées au point de vue rémanence et coercivité, à un

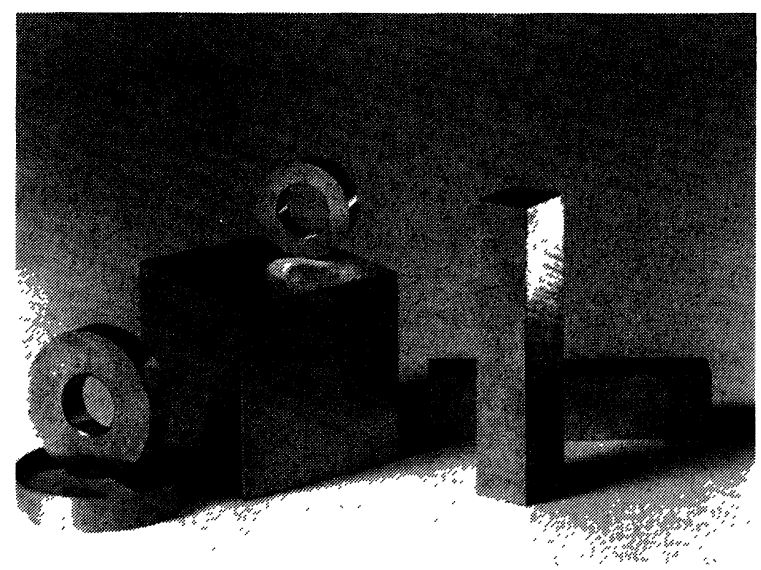

FIG. 9. - Exemples de pièces réalisées en Ticonal 2000 à haute force coercitive. Le cube central a une arête de quatre centimètres et pèse cinq cents grammes.

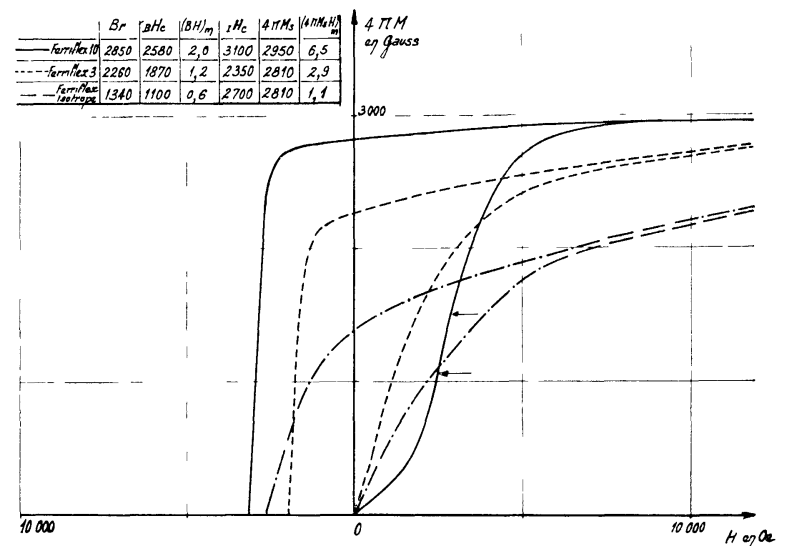

FIG. 10. - Courbes de première aimantation et de désaimantation de diverses qualités d'élastomères magnétiques. Noter les grandes différences que procurent de meilleurs séparations et alignements des grains de ferrite. 
point tel que dans la direction perpendiculaire au champ orienteur, la pente à l'origine est énorme et le point d'inflexion a disparu. Après revenu, dont l'effet est de mieux différencier les phases en présence en entraînant une légère diminution des aimantations à saturation et rémanente, la forme de chaînes de sphères disparaît au profit de celle d'ellipsoïdes allongées : la coercivité et le facteur de mérite doublent, les pentes à l'origine, à la rémanence et au recul décroissent. Une cristallisation orientée permettrait d'exalter encore plus ces valeurs.

Avec comme point de départ le Ticonal à 600 Oe de coercivité, ces valeurs élevées de coercivité n'ont pu être obtenues qu'au prix d'une forte élévation de la teneur en cobalt ( $38 \%$ contre $24 \%$ en poids), d'une addition importante de titane ( $8 \%$ en poids) et d'un traitement thermique brutal, consistant en une trempe après homogénéisation et après maintien sous champ [30]. Le rôle du titane n'est pas encore bien élucidé ; il se pourrait qu'il réside dans la belle régularité des particules obtenues [31], [32], la nette séparation d'aimantation entre les deux phases [33] qui superpose peut-être à l'anisotropie de forme une anisotropie de surface [34], en même temps qu'une distorsion tétragonale le long de l'axe (100) [31] induirait une anisotropie cristalline importante, décalant ainsi vers de plus hautes coercivités le retournement par « curling » [35].

Cette progression vers les hautes coercivités est le résultat d'une longue succession de travaux de laboratoires et les matériaux correspondants sont maintenant produits industriellement. L'une des difficultés importantes à résoudre consistait dans l'excessive fragilité d'un alliage à petits grains, soumis à des traitements énergiques. La difficulté est maintenant tournée et la figure 9 montre quelques réalisations faites couramment en Ticonal 2000 [14]: bagues de focalisation pour tubes à ondes progressives, cube et barreaux pour moteurs linéaires. Le cube pèse environ un demi-kilogramme et est exempt de tout défaut habituellement rencontré dans ce type de pièces, soufflures de coulées et tapures de traitements thermiques.

S'il semble encore possible d'améliorer ces alliages [31] pour les porter à des coercivités de 5000 Oe et des facteurs de mérite de 30 à $35 \times 10^{6} \mathrm{G}$.Oe, il est hors de doute que les progrès futurs seront très lents et très coûteux. On pourrait donc penser que le matériau Ticonal s'effacera progressivement dans l'avenir devant des matériaux plus coercitifs comme les ferrites ou des matériaux à la fois plus coercitifs et aussi rémanents comme les cobalt-terres rares. Effectivement, la production des Ticonals a suivi une chute importante vers le milieu des années 60 [8], avec la montée progressive des ferrites. Mais elle a maintenant retrouvé son niveau antérieur et l'on peut considérer que le palier atteint se maintiendra encore de nombreuses années. Plusieurs raisons militent pour cette stabilité. Obtenus par les techniques de fonderie, ils ne nécessitent que des outillages sommaires bien adaptés aux petites séries, tout en assurant un bon respect des tolérances ; à l'opposé, les aimants préparés par voie des poudres (Ticonals frittés, ferrites, cobalt-terres rares) demandent des outillages précis, complexes et coûteux, justiciables de séries importantes, ou font appel à des techniques d'usinage onéreuses. En deuxième lieu, leur forte rémanence (de 8 à $13000 \mathrm{G}$ ) est très utile lorsque le flux demandé ne peut être fourni que dans une faible section. En dernier, c'est leur excellente stabilité thermomagnétique $(\Delta B / B=-0,01$ à $0,04 \% \quad C)$ jusqu'à des températures élevées $\left(500^{\circ} \mathrm{C}\right)$ qui impose leur emploi dans les instruments de mesure et les systèmes où la stabilité thermique est capitale; dans le domaine de l'électronique professionnelle, ce sont surtout les carcinotrons type $\mathbf{M}$ à champs croisés, les isolateurs à déplacement de champ, les limiteurs ou doubleurs de fréquence, les résonateurs à monocristaux de grenats, qui font et feront toujours appel à ce type de matériau. Dans d'autres dispositifs, comme les magnétrons, les amplificateurs à champs croisés, les klystrons amplificateurs à plusieurs cavités, les carcinotrons de type $\mathrm{O}$, les tubes à ondes progressives, les isolateurs à résonance gyromagnétique, les circulateurs à jonction, il est possible que d'autres matériaux, comme les aimants cobalt-terres rares se substituent peu à peu aux Ticonals à haute force coercitive, les conditions de stabilité thermique y étant moins critiques $[36,37]$.

4. Le caoutchouc magnétique orienté. - L'idée d'aimants permanents basés sur le principe de la particule monodomaine a été particulièrement féconde puisqu'elle a conduit aux aimants en poudres, de fer ou de fer cobalt, aux Ticonals et aux bandes magnétiques. Dans ces trois cas, l'anisotropie uniaxe recherchée est obtenue par la forme allongée des particules ferromagnétiques. Une tout autre possibilité a été offerte en 1942, lorsqu'il fut découvert [38] que de fines poudres de manganèse-bismuth possédaient la coercivité intrinsèque énorme de $12000 \mathrm{Oe}$, pour une taille voisine de trois microns. Dans ce matériau et ses semblables, la coercivité a pour origine la forte anisotropie magnéto-cristalline qui ancre la direction d'aimantation sur une direction privilégiée du réseau cristallin; à l'opposé de l'anisotropie de forme, où le compactage au-delà de $66 \%$ fait décroître la coercivité, l'anisotropie cristalline permet d'atteindre la densité maximum et une rémanence accrue, sans perte de coercivité. Pour des raisons de stabilité temporelle, ces matériaux à base de manganèse et de métaux oxydables n'ont jamais atteint le stade industriel.

Mais l'idée fut reprise et appliquée [39] aux ferrites de baryum, strontium et plomb, connues depuis bien longtemps [40], en donnant naissance à la classe des aimants ferrites qui occupent $50 \%$ du marché mondial (Fig. 3) ; l'introduction d'oxyde de baryum, strontium ou plomb dans le réseau orthorhombique de 
l'oxyde antiferromagnétique $\alpha-\mathrm{Fe}_{2} \mathrm{O}_{3}$ a pour effet de différencier les deux sous-réseaux magnétiques pour donner naissance à un ferrimagnétique, et le passage à une structure hexagonale s'accompagne de l'apparition d'une forte anisotropie uniaxiale. Nous ne nous attarderons pas sur les ferrites frittées, dont la technologie, les performances et propriétés ont déjà été bien décrites par ailleurs [41], pour ne nous consacrer qu'au caoutchouc magnétique orienté, qui connaît un développement considérable (Fig. 3). A l'origine se trouve un décret de la législation américaine obligeant les fabricants de frigidaires à délivrer des appareils s'ouvrant facilement de l'intérieur. Une bande aimantée et enfermée dans un joint d'étanchéité fournit une solution élégante, la grande surface de contact autorisant l'emploi d'un matériau à faible
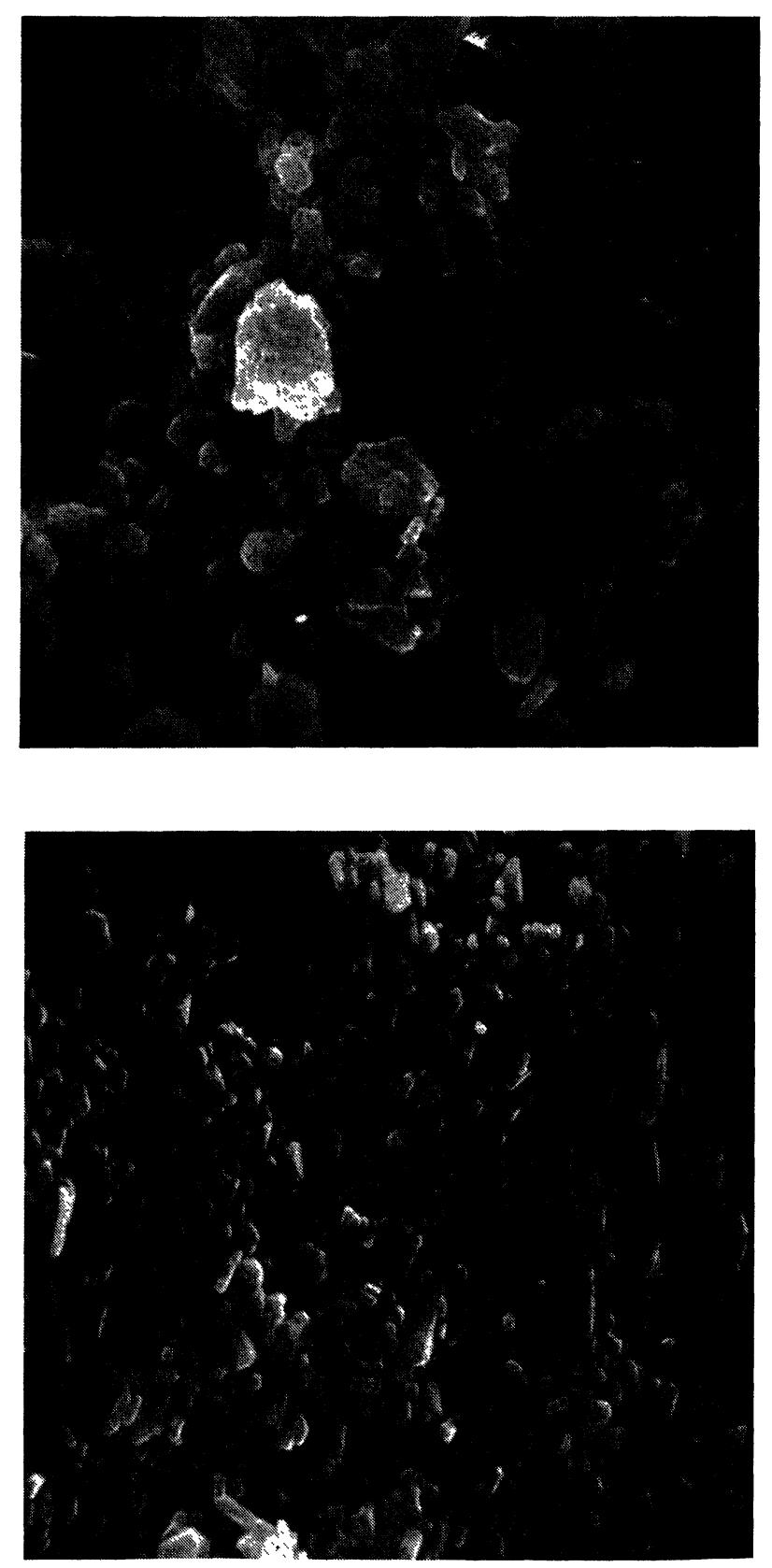

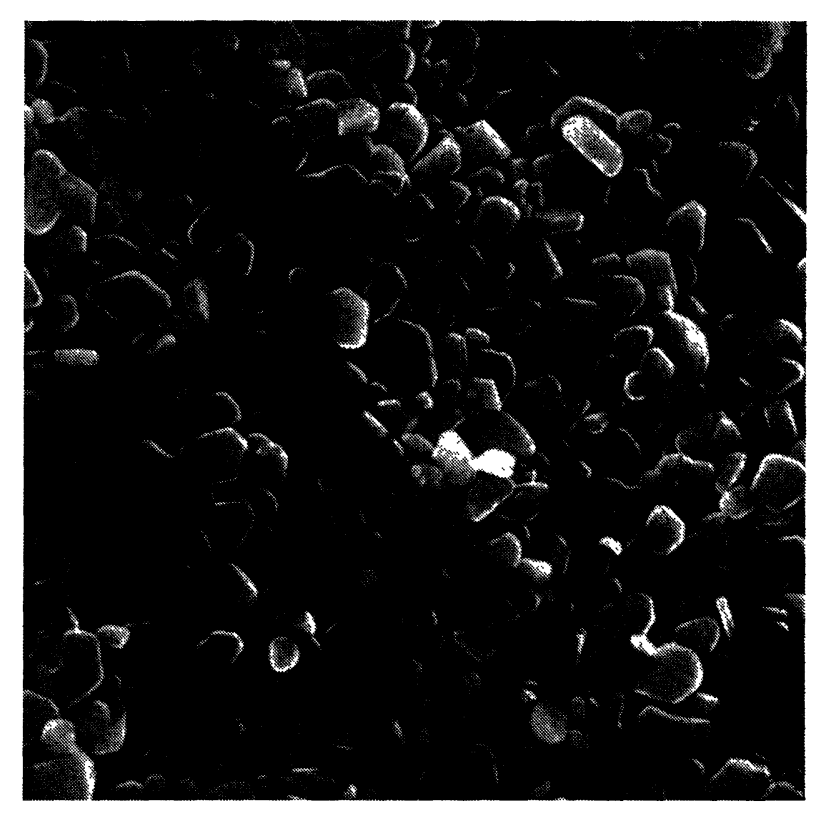

rémanence. La coercivité doit être cependant importante pour permettre à l'aimant de résister aux actions démagnétisantes provoquées par l'ouverture infiniment répétée de la porte. De la poudre de ferrite, enrobée à $65 \%$ en volume dans un élastomère et extrudée fournit la solution adéquate. Les caractéristiques magnétiques en sont illustrées sur la figure 9 , sous le nom de Ferriflex [14] isotrope. La première aimantation croît régulièrement, avec un accident de pente à peine perceptible vers $2500 \mathrm{Oe}$; la saturation est difficile à obtenir et la rémanence est égale à la moitié de l'aimantation à saturation, valeur caractéristique des systèmes uniaxes sans orientation préférentielle.

Ce matériau souple apporte une qualité mécanique importante que ne possèdent pas les autres matériaux

FIG. 11. - Examens au microscope à balayage de poudres de ferrites. En haut, une poudre destinée au caoutchouc magnétique isotrope est constituée d'agrégats solides de grains monodomaines. En bas, la poudre obtenue par voie chimique est constituée de grains séparés, et homogènes en taille. A droite, la même poudre, enrobée de caoutchouc, s'est alignée mécaniquement par calandrage selon le plan de la feuille. 
à aimants, à savoir la facilité de mise en forme, sans usinages délicats et coûteux. Aussi, n'est-il pas étonnant qu'on est recherché à atteindre d'autres marchés, comme l'attraction décorative ou fonctionnelle, les volants magnétiques, les petits moteurs à courant continu,... etc., en augmentant les performances magnétiques nécessaires à l'emploi sous de petites dimensions. Une calcination à température plus haute, suivie d'un broyage, autorise la libération de grains de ferrite séparés, dont la forme en plaquettes se prête bien à une orientation mécanique par calandrage, donnant naissance à la qualité Ferriflex 3 [14] à $(B H)_{\max }=1,2 \times 10^{6} \mathrm{G}$.Oe, soit le double du Ferriflex isotrope. Mais le broyage induit des dislocations de réseau, conduisant à une première aimantation (Fig. 10) par déplacement de parois, et une baisse de coercivité par nucléation aisée de domaines inverses. Aussi l'avenir appartient-il à des poudres de ferrite obtenues sans broyage, par voie chimique, et correctement alignées par calandrage et champ magnétique [42], conduisant en laboratoire à la performance exceptionnelle de $(B H)_{\max }=2,0 \times 10^{6}$ G.Oe. La différence de qualité des poudres apparaît nettement sur les clichés de microscopie électronique à balayage (Fig. 11). Une poudre non broyée est faite d'agglomérats de grains frittés, inaptes à l'orientation mécanique; le broyage libère les grains, mais détruit statistiquement leur forme avantageuse de plaquettes ayant grossi préférentiellement selon le plan perpendiculaire à l'axe d'anisotropie. Quant aux poudres obtenues par voie chimique, elles montrent une très belle homogénéité, une forme régulière, et l'absence totale d'agrégats. L'orientation mécanique est aisée et se suit facilement aux rayons $X$ (Fig. 12), les raies

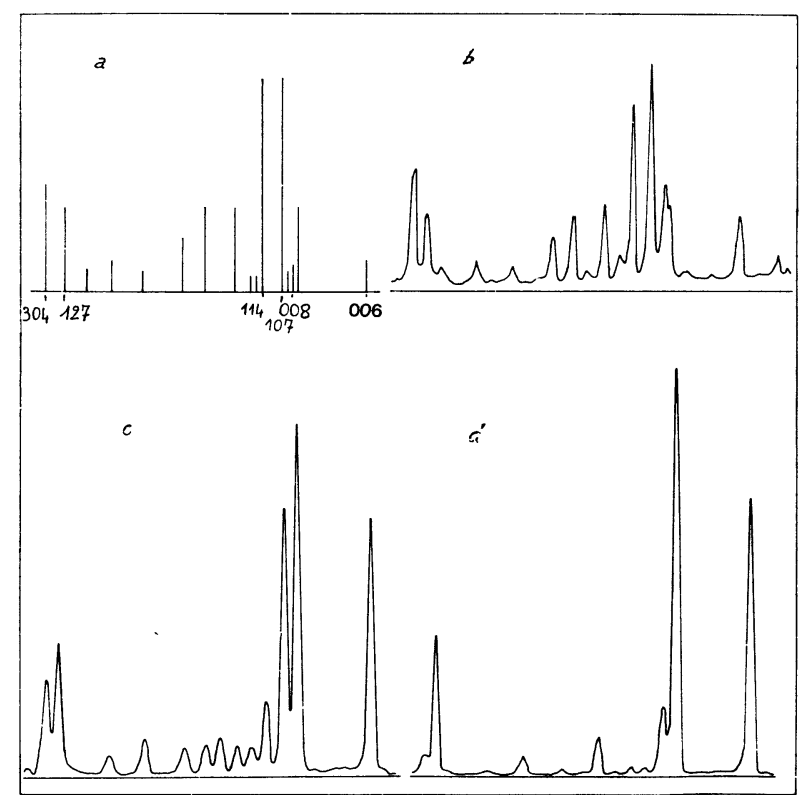

FIG. 12. - Examens aux rayons $\mathrm{X}$ de la surface de caoutchoucs magnétiques : $a$ ) Spectre isotrope théorique. $b$ ) Spectre de Ferriflex isotrope, à poudre agglomérée et extrudée. c) Spectre de Ferriflex 3, à poudre broyée et calandrée. d) Spectre de Ferriflex 10, à poudre obtenue chimiquement, calandrée et alignée magnétiquement.
(001) étant d'autant plus favorisées que l'orientation est meilleure comme lors du frittage [43] ; dans cette direction, les propriétés magnétiques sont fortement accrues, au détriment des propriétés dans le plan de la feuille.

L'apparition de ces qualités hautement performantes peut changer considérablement la conception et la fabrication des circuits magnétiques. Par exemple, dans les haut-parleurs, la source de champ est un aimant cylindrique, soit en Ticonal de grande longueur, soit en ferrite frittée de grande section. La membrane oscillante, de forme conique, n'est actionnée que sur une section circulaire étroite, ce qui entraîne une distorsion acoustique importante aux basses fréquences. Une idée toute nouvelle a été celle du hautparleur plat [44], où une lame conductrice en zig-zag actionne la totalité de la membrane plane (Fig. 13).
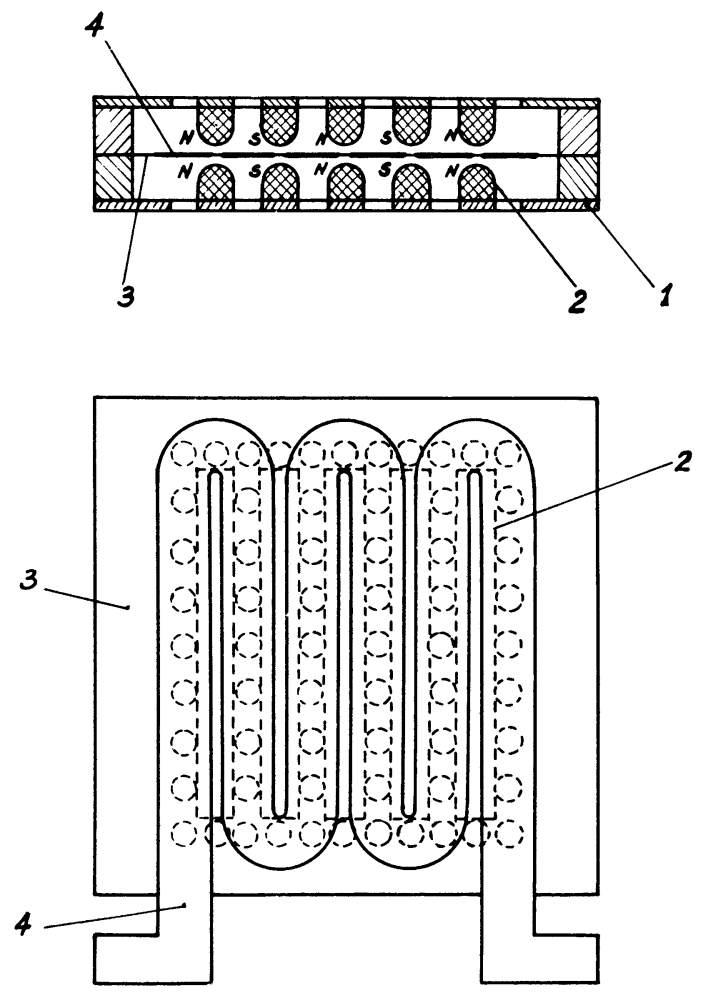

FIG. 13. - Principe du haut-parleur plat, d'après [44] et [45]. 1) Plaque polaire en fer doux. 2) Aimant. 3) Membrane. 4) Conducteur.

Le champ nécessaire au mouvement est fourni par des barreaux de ferrite frittée, et la surface active compense largement les pertes de rendement électrodynamique, au profit d'une réponse acoustique excellente. Ce dispositif n'a pas connu le succès attendu, le collage de barreaux unitaires sur une plaque support étant onéreux en main-d'œuvre. Avec le caoutchouc magnétique à qualité supérieure, l'aimant est réalisé en une seule pièce par estampage de feuilles plastiques magnétiques [45]. Il est probable que d'autres applications intéressantes du même ordre verront le jour dans un proche avenir, comme par 
exemple la fixation rapide des clichés sur les rotatives d'imprimerie.

5. Les aimants cobalt-terres rares. - Comme ces matériaux sont relativement nouveaux et font l'objet d'études intensives, nous nous attarderons plus longuement sur leurs structures et propriétés, dont la connaissance est indispensable pour la compréhension de leurs performances et l'estimation de leur valeur potentielle future.

A partir des années 60 , grâce à la mise à leur disposition des éléments de terres rares en quantité importante et de grande pureté, de nombreux laboratoires se sont intéressés à l'étude de leurs alliages, par la détermination de leurs diagrammes d'équilibre, de leurs structures cristallines et de leurs propriétés physiques [46]. Leur structure électronique pouvait laisser prévoir un intérêt important des terres rares dans le domaine des aimants permanents : une couche $4 \mathrm{f}$ profonde et bien protégée va contenir un nombre croissant d'électrons non appariés, d'où un fort moment de spin, auquel s'ajoutera ou se retranchera un fort moment orbital. Par ailleurs, un couplage spin-orbite important assure une forte interaction entre moment magnétique et réseau, et laisse prévoir une grande anisotropie magnéto-cristalline. Malheureusement, cette localisation interne des moments fait que leurs interactions mutuelles sont faibles, et les températures d'ordre, basses : la plus élevée est celle du gadolinium métallique où la température de Curie n'est que de $290 \mathrm{~K}$. Or, on exige d'un matériau à aimant permanent que sa température de Curie soit au moins supérieure à $300^{\circ} \mathrm{C}$, la quasi-totalité des applications se faisant à l'ambiante et au-dessus. Pour profiter des propriétés spéciales des terres rares, il faut donc les allier à d'autres métaux ferromagnétiques possédant un point de Curie élevé et un moment magnétique important, ce qui focalise sur le fer, le nickel et le cobalt [47]. Les terres rares-fer sont difficiles à préparer, la grosse taille de l'atome de fer rendant les structures intéressantes instables. Par ailleurs, l'introduction d'électrons supplémentaires dans la bande de conduction diminue le moment intrinsèque du métal de transition; ceci exclut les terres raresnickel, qui ont aussi de faibles points de Curie.

La famille intéressante se limite donc aux terres rares-cobalt [3], et plus spécialement aux alliages de samarium et de cobalt, dont un diagramme schématique est donné sur la figure 14 (pour une revue générale des composés terres-rares-autres métaux, voir [48]). Il ne s'agit pas à proprement parler d'alliages, mais de composés intermétalliques définis, où la terre rare permet au cobalt de s'ordonner suivant des structures uniaxiales différentes de celle du cobalt métallique, avec quelquefois des distances interatomiques bien plus courtes que dans le métal pur [49], autorisant ainsi de fortes interactions d'échange et de grandes anisotropies cristallines. Une autre singularité de ces matériaux est la possibilité de nombreuses substitutions désordonnées ou ordonnées, autorisant de larges domaines d'homogénéité pour les 1-5 et les 2-17, et des transitions aisées d'un composé
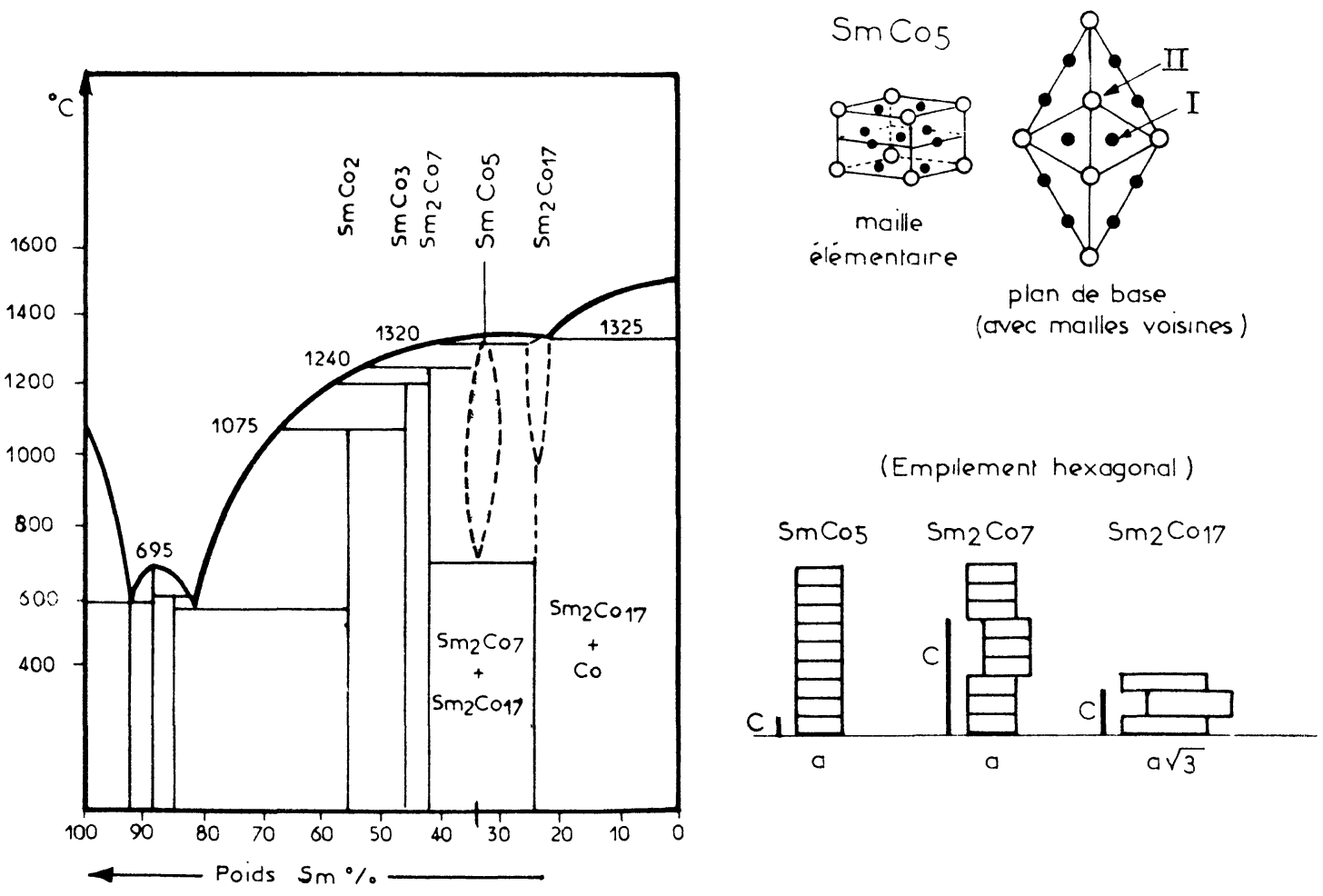

Fig. 14. - Diagramme de phases samarium-cobalt.

Structures cristallines schématiques des composés $\mathrm{SmCo}_{5}, \mathrm{Sm}_{2} \mathrm{Co}_{7}$ et $\mathrm{Sm}_{2} \mathrm{Co}_{17}$. 
à l'autre. La structure de base est l'hexagonal $\mathrm{CaCu}_{5}$ (Fig. 14) ; le remplacement d'un cobalt du plan de base par une terre rare déplace la structure vers les composés 2-7 ou 5-19 [50] selon leur périodicité, alors que le remplacement d'une terre rare par une paire d'atomes de cobalt fait glisser vers la structure 2-17. Cette originalité conduit à rendre délicate la maîtrise de la fabrication de ces matériaux, d'autant plus que l'optimum des propriétés magnétiques est atteint [51] pour une composition bien définie, intermédiaire entre les phases $1-5$ et $2-7$ ou $5-19$, et que les terres rares sont très sensibles à l'oxydation à haute température. La figure 15 illustre bien cette

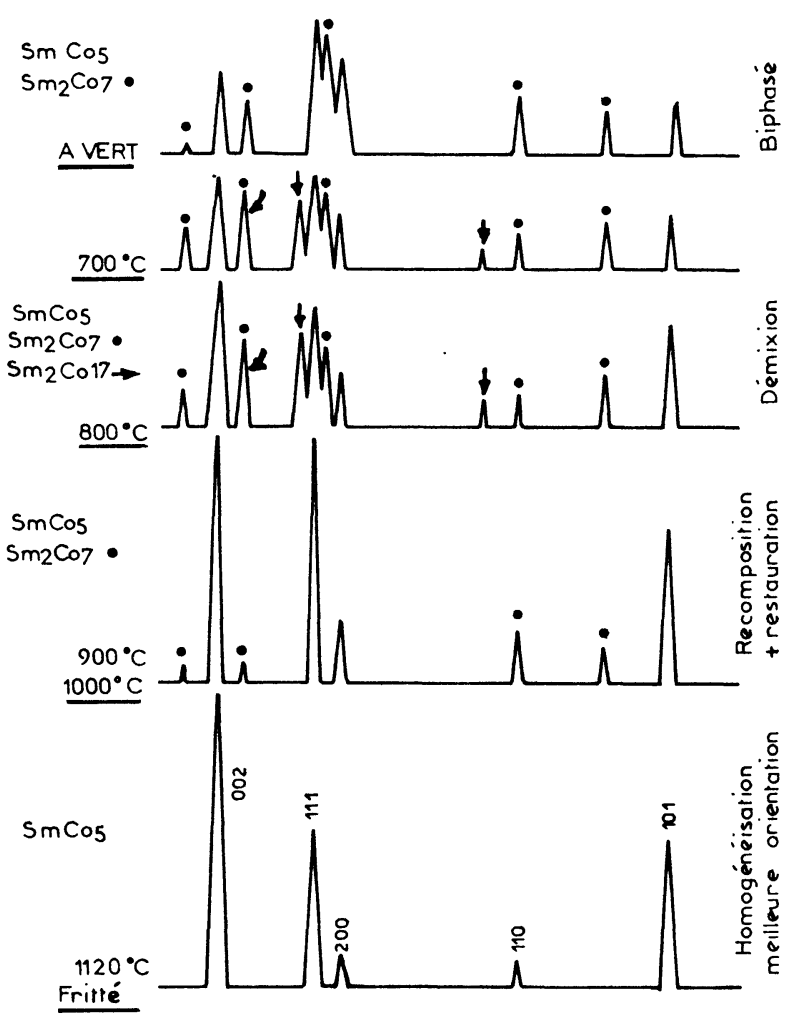

FIG. 15. - Evolution du diagramme de rayons $\mathrm{X}$ lors du frittage de comprimés de poudre cobalt-samarium.

complexité ; dans cette expérience, on a suivi l'évolution du spectre de rayons $\mathrm{X}$ de comprimés d'alliage, lors du frittage [52]. A température ambiante, ne sont détectables que les raies des composés 1-5 et 2-7. A température montante, les raies du 2-7 croissent en importance en même temps qu'apparaissent celles du 2-17 ; cette mixture d'états à la fois réduit et oxydé proviendrait d'une oxydation préférentielle du samarium par l'oxygène contenu dans les pores du comprimé, à laquelle se superposerait une démixtion $[53,54]$ de la phase 1-5 en ses deux proches voisins. La phase 15 ne serait donc pas stable à l'ambiante (Fig. 14) et ne pourrait être obtenue que par trempe rapide. Signalons que cette démixtion est contestée [50], les caractères distinctifs de ces phases n'étant observés qu'après vieillissement prolongé. A haute tempé- rature, les phases voisines disparaissent aux rayons $\mathrm{X}$ pour ne laisser place après frittage qu'à un spectre caractéristique de la structure de base $\mathrm{CaCu}_{5}$; la phase 2-7 subsisterait partiellement, mais dans un état très désordonné [55].

Les courbes de première aimantation et de désaimantation présentent des caractéristiques totalement différentes de celles décrites plus haut pour le PlatineCobalt, Ticonal et caoutchouc orienté. Les courbes de la figure 16 ont été obtenues en saturant progressivement un échantillon fritté dans un champ continu,

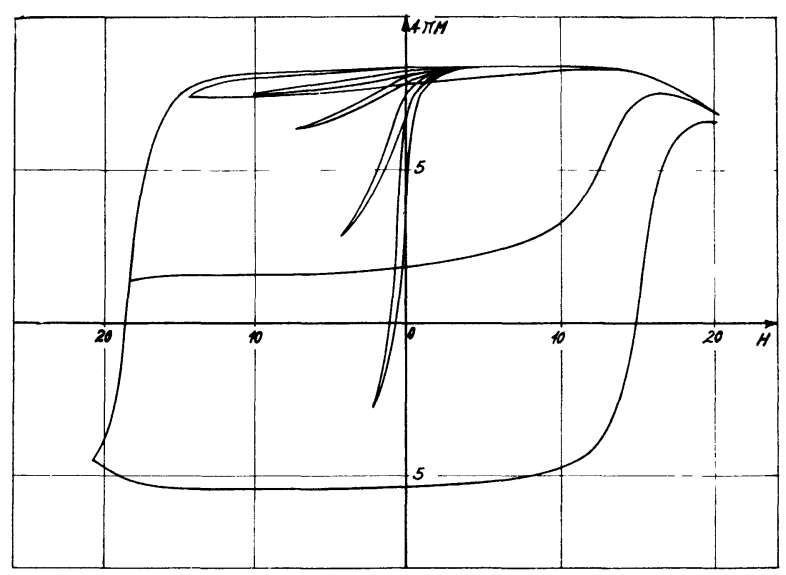

Fig. 16. - Cycles d'hystérésis progressive d'un échantillon de samarium cobalt fritté

La chute de l'aimantation vers $20 \mathrm{kOe}$ est un artefact dû à la saturation des pièces polaires.

alternativement positif et négatif et de valeur croissante. Le déplacement des cycles d'hystérésis vers les aimantations positives traduit la présence d'une aimantation résiduelle importante que n'arrive pas à retourner un champ inverse égal au champ de saturation : la coercivité des grains croît avec le champ appliqué, et même pour de grandes valeurs de champ, le cycle d'hystérésis ne retrouve pas la symétrie par rapport à l'origine qui caractérisait les autres matériaux (Fig. 6 et 10). Il s'agit donc d'un mécanisme entièrement nouveau, dont l'approfondissement fait l'objet de multiples études, tant sa compréhension est vitale pour le développement des futurs matériaux [56]. En première approximation, on peut dire que ces matériaux à forte anisotropie cristalline satisfont au paradoxe de Brown [25] d'une coercivité indépendante de la taille du cristal. Le retournement de l'aimantation ne peut se faire que par rotation cohérente des moments, au moins en un point du cristal, et la coercivité théorique est égale au champ d'anisotropie, de 2 à $3 \times 10^{5}$ Oe. Mais de telles valeurs n'ont pas encore été atteintes, ce que l'on explique par la présence de défauts, essentiellement superficiels, retenant de petits domaines non retournés lors de la première aimantation $[23,24]$, et qui jouent le rôle de sites actifs pour la nucléation d'une aimantation inverse lors du retournement du champ [26]. 


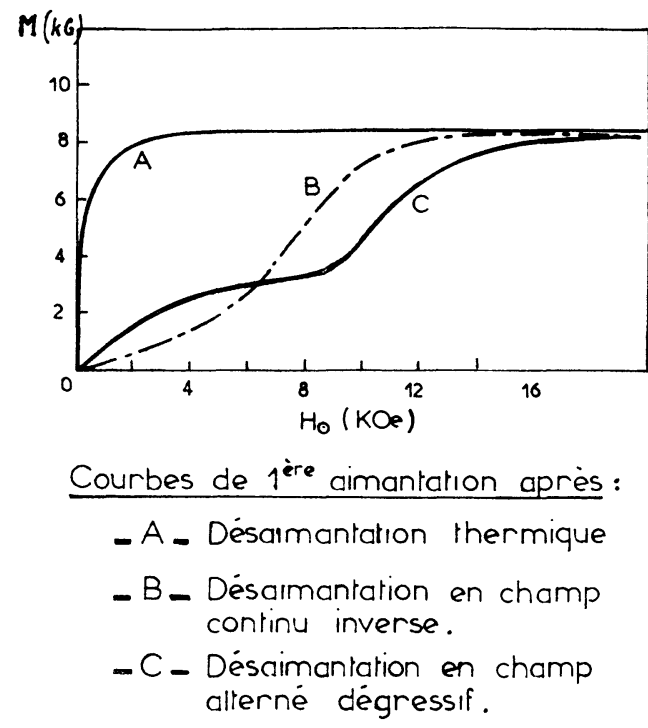

Configurations initiales.

A
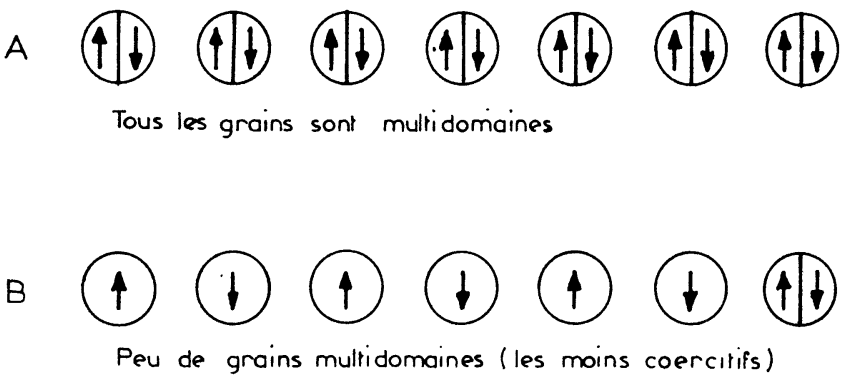

Inversion de $50 \%$ des aurres.

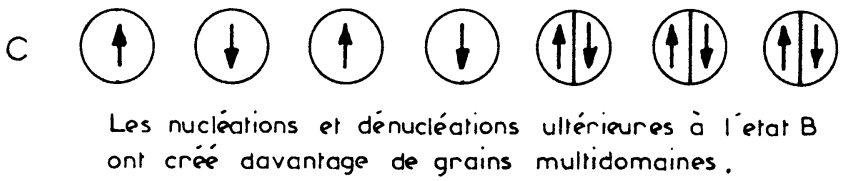

Fig. 17. - Allure des courbes de première aimantation du samarium-cobalt en fonction du mode choisi pour désaimanter l'échantillon : thermiquement, par champ continu inverse, par champ alternatif décroissant.

La nature exacte de ces défauts n'a pas encore été bien déterminée. Comme pour les ferrites dures [5759], cette structure particulière va modifier profondément l'allure des courbes de première aimantation, selon la technique utilisée pour désaimanter l'échantillon (Fig. 17) [27]. En l'état désaimanté thermiquement, chaque grain est un polydomaine, dont l'aimantation se fait par déplacement de parois à très faible coercivité : la saturation est immédiatement atteinte, avec une pente approximativement égale au facteur démagnétisant de la sphère. Par désaimantation en champ continu inverse est générée une structure à aimantation macroscopique nulle, mais constituée en fait d'un peu de grains en polydomaines, la quasi-totalité de l'échantillon étant répartie en deux familles de grains d'aimantation opposée. Il faut atteindre le champ de nucléation du déplacement des parois [56] pour retourner la moitié de ces grains et faire croître rapidement l'aimantation. Par désaimantation en champ alternatif, on augmente simplement la quantité de grains à structure polydomaine, et l'aimantation initiale croît un peu plus rapidement. Ces structures sont très bien observées en lumière polarisée [60] et démontrent une fois de plus la quasi-indépendance des grains dans les aimants frittés [27].

Sur le plan des applications, les matériaux à base de terres rares se comportent comme des aimants idéaux [41]. La rectangularité de leur cycle d'hystérésis leur assure les propriétés optima, à savoir des énergies spécifiques et de recul égales au quart du carré de l'aimantation à saturation, et une résistance à la désaimantation égale au champ coercitif intrinsèque. Lorsqu'auront été mises en place les structures industrielles adéquates pour la production de masse, ils supplanteront tous leurs concurrents dans les appli- cations où l'encombrement, l'épaisseur, la résistance magnétique sont prépondérantes, pour un coût donné. Ils sont également idéaux sur le plan du calcul des circuits magnétiques, l'approximation de l'aimantation uniforme s'y appliquant particulièrement bien. Comme il est connu [61] cette approximation permet de traiter un cylindre aimanté, soit comme une répartition de masses magnétiques sur les faces terminales, soit comme un courant de surface sur les faces latérales, en négligeant les gradients volumiques. Ces deux types d'approche ont été confrontés à l'expérience pour le calcul de la force entre aimants en répulsion [62] et du champ axial créé par une bague [37] ; la concordance expérience-calcul est excellente (Fig. 18 et 19). On notera particulièrement que si les forces de répulsion au contact doivent être, pour différents matériaux, comme les rapports des coercivités d'induction [63], elles varient plutôt, en présence d'un entrefer, comme le carré des coercivités intrinsèques. Dans cette application à laquelle certains songent pour la lévitation des véhicules à grande vitesse [64], les aimants cobalt-terres rares sont remarquables en arrivant à léviter 60 fois leur poids en répulsion et 200 fois en attraction [37].

$\mathrm{Si}$, sur le plan magnétique, les aimants cobaltterres rares surclassent largement leurs concurrents, il ne faut pas oublier que les propriétés magnétiques ne sont pas les seules retenues par les utilisateurs qui accordent aussi beaucoup d'attention aux propriétés mécaniques, à la mise en forme magnétique, à la stabilité thermique et au coût. La voie purement métallurgique [65] étant maintenant abandonnée, compte tenu des difficultés d'élaboration, les techniques de production s'orientent vers la production de masse par la métallurgie des poudres, comme pour 

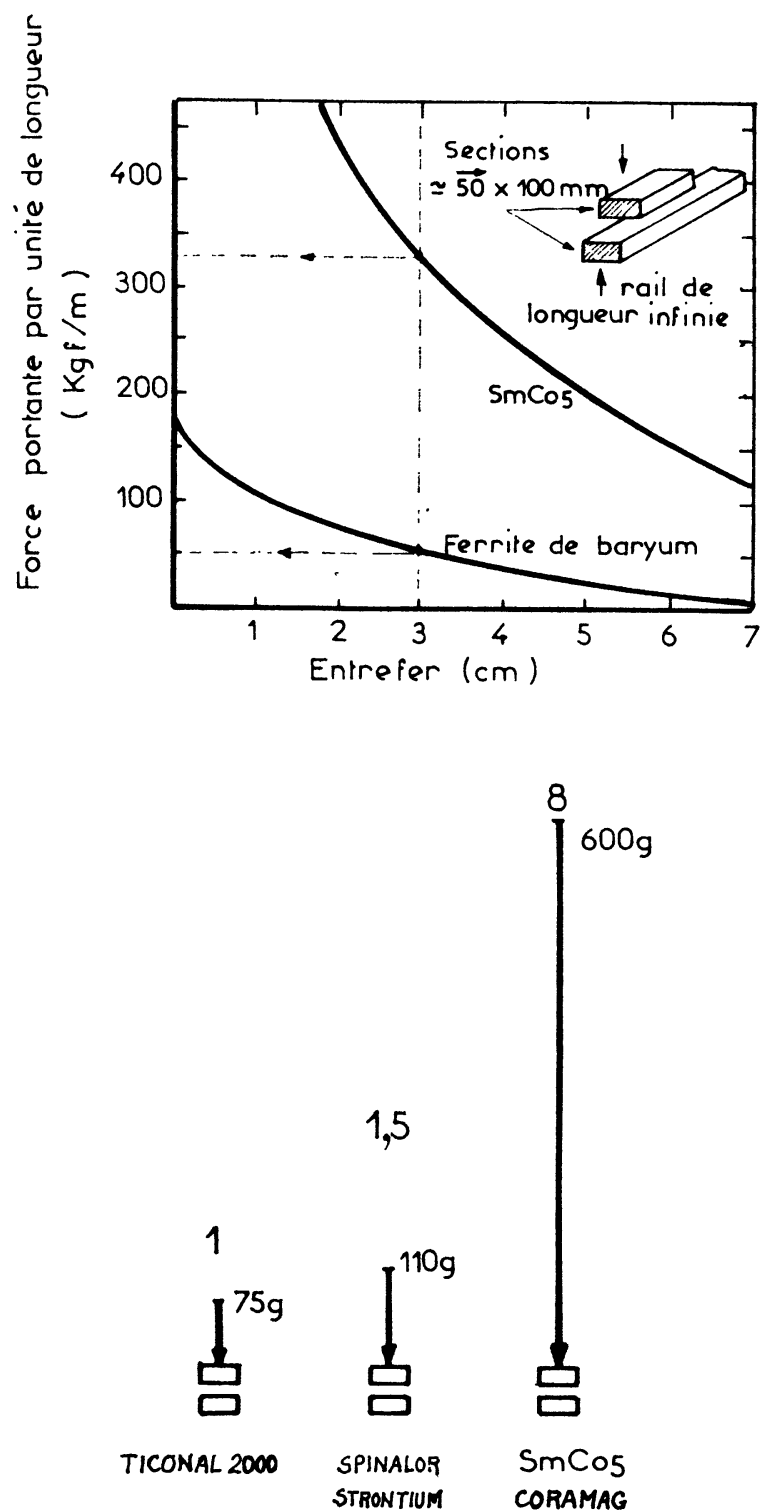

Fig. 18. - Calcul de la force de répulsion entre aimants, d'après [62] et vérification expérimentale pour des matériaux à haute force coercitive (approximation des masses magnétiques superficielles). a) Comparaison des forces de répulsions segment/rail entre $\mathrm{SmCo}_{5}$ et le ferrite de baryum. b) Comparaison entre aimants de $\varphi=14$, épaisseur 7 pour un entrefer de $2,5 \mathrm{~mm}$.

les ferrites [41]. Quant aux champs de saturation nécessaires, ils s'écartent notablement de la vieille règle empirique utilisée pour les autres matériaux [66] (soit cinq à six fois le champ coercitif intrinsèque) et ne nécessitent pas les courants supraconducteurs, comme annoncé au début. Il suffit d'un champ capable de déplacer les parois et de décrocher des défauts les reliquats de domaines inverses; un champ de 15 à $20000 \mathrm{Oe}$, d'autant plus faible que l'orientation est meilleure, est suffisant pour atteindre $95 \%$ des propriétés magnétiques (Fig. 20) ; on notera que la courbe de la coercivité intrinsèque coupe la droite $B / H=1$, ce qui implique que certains grains ont une coercivité supérieure au champ appliqué et interdit la désai-

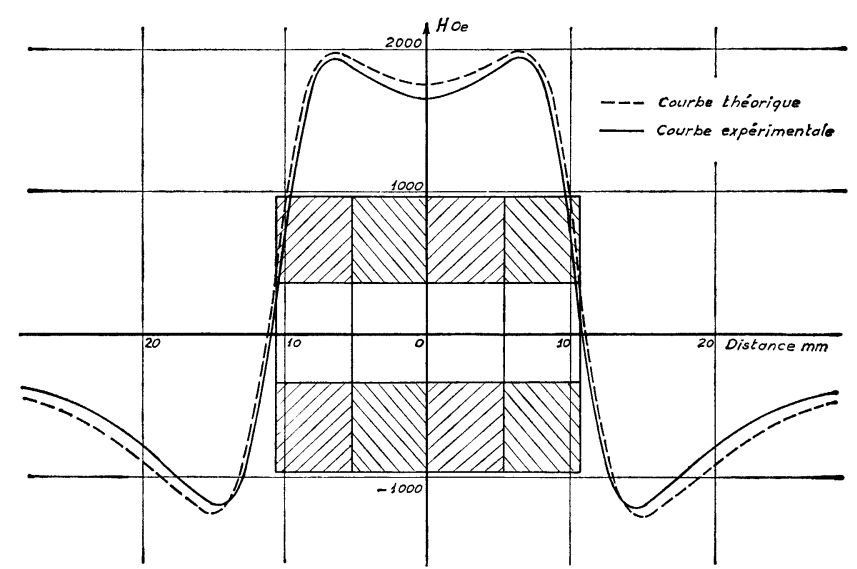

FIG. 19. - Calcul du champ axial d'une bague et vérification expérimentale pour le Coramag [14] (approximation des courants de surface).

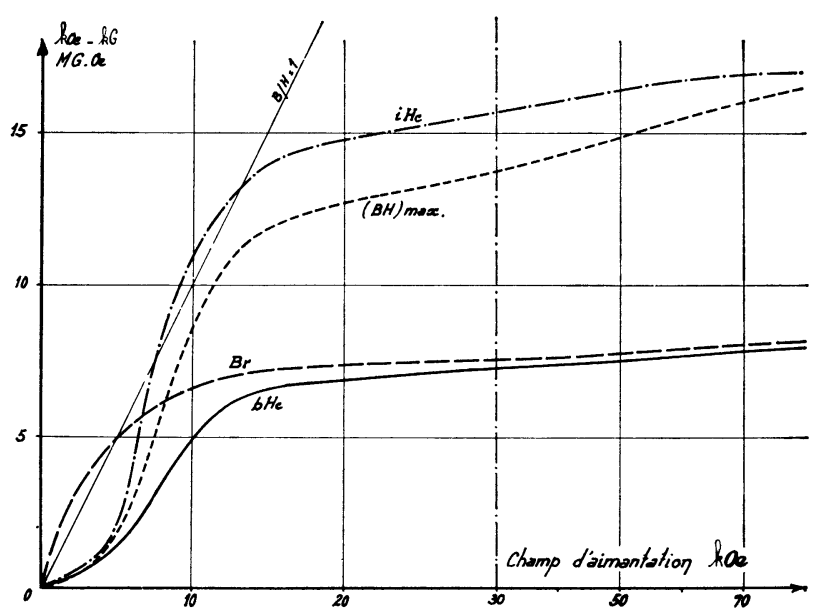

Fig. 20. - Variation des propriétés magnétiques du Coramag [14] avec le champ de saturation appliqué sur un échantillon ayant un taux d'orientation égal à $85 \%$.

mantation totale par champ alternatif oscillant. En ce qui concerne la stabilité thermique, on distingue habituellement entre : - les pertes magnétiques réversibles, liées à la variation de l'aimantation à saturation - les pertes magnétiques irréversibles et récupérables par réaimantation, liées à la variation de coercivité et à l'existence d'un champ démagnétisant interne plus ou moins intense selon le point de travail les pertes magnétiques irréversibles et irrécupérables par réaimantation, liées à des transformations métallurgiques. La figure 21 donne les courbes de désaimantation mesurées sur un cylindre en circuit ouvert à $B / H=1$, après passage d'une heure à l'air aux températures indiquées. Jusqu'à $300^{\circ} \mathrm{C}$, la stabilité est excellente. Au-delà, à $340^{\circ} \mathrm{C}$, la courbe indique une perte magnétique toutefois récupérable par réaimantation, et due à la proximité du point d'annulation du champ coercitif intrinsèque [67]. Ce point coïncide avec la température de Curie du composé $\mathrm{Sm}_{2} \mathrm{Co}_{7}\left(T_{\mathrm{c}}=410^{\circ} \mathrm{C}\right)$ et non pas avec la température 


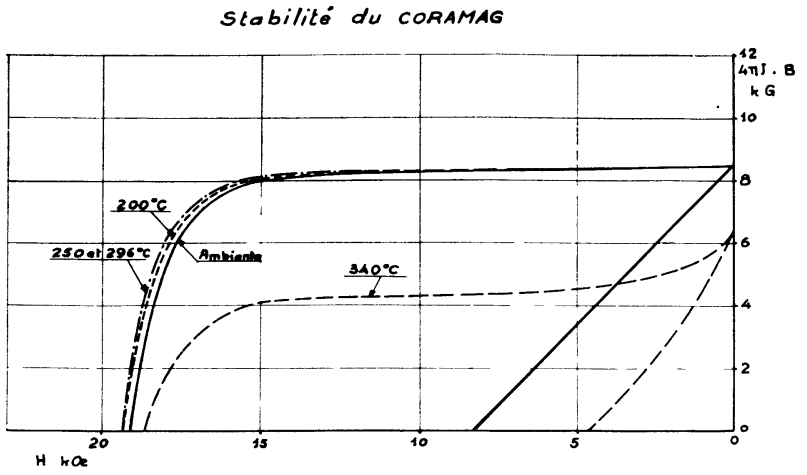

Fig. 21. - Courbes de désaimantation d'un cylindre de Coramag [14], de perméance unité, après passage à l'air, pour une heure à différentes températures.

de Curie de la matrice $\mathrm{SmCo}_{5}\left(T_{\mathrm{c}}=720^{\circ} \mathrm{C}\right)$, démontrant ainsi l'importance de la phase 2-7 ou d'une phase voisine [68], ordonnée ou non, pour le mécanisme de nucléation de domaines inverses. A partir de $400^{\circ} \mathrm{C}$, et pour des temps de maintien courts, les matériaux cobalt-terres rares montrent des pertes de champ coercitif irrécupérables par réaimantation, et induites certainement par une transformation métallurgique; celle-ci serait la démixtion en 2-7 et 2-17 [69,53-54], mais ces phases ne sont bien visibles qu'après des maintiens très prolongés et cette démixtion est contestée, au moins pour les terres rares
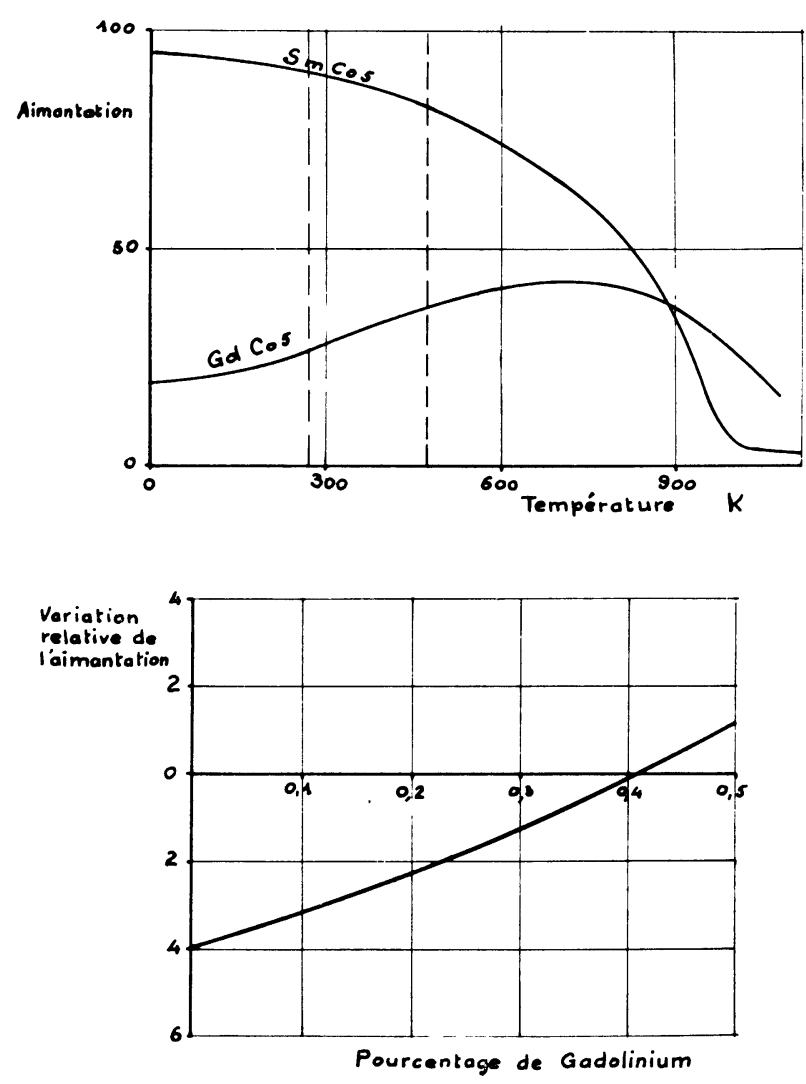

Fig. 22. - En haut, variations de l'aimantation de $\mathrm{SmCo}_{5}$ et $\mathrm{GdCo}_{5}$, avec la température d'après [70]. En bas, pertes réversibles d'un mélange $\mathrm{Sm}_{1-x} \mathrm{Gd}_{x} \mathrm{Co}_{5}$, d'après [72]. apparentées au samarium [50]. Quant aux pertes réversibles, elles n'atteignent que $0,04 \%$ par degré, un peu plus fortes que pour les Ticonals $(0,02 \%)$ mais bien inférieures aux ferrites $(0,2 \%)$. Elles sont d'ailleurs quasi contrôlables à volonté au prix d'une baisse des propriétés, par un choix judicieux d'un mélange de terres rares, dont les variations de l'aimantation avec la température peuvent se compenser dans un certain domaine (Fig. 22).

En conclusion, les matériaux de base de terres rares, par leurs performances et leur variété, contribueront certainement au développement prodigieux que connaissent les aimants.

6. Conclusion. - Au terme de ce large tour d'horizon, il nous paraît intéressant de dresser quelques perspectives sur l'avenir des matériaux à aimants et de leur industrie. Pour les matériaux, le virage en qualité, annoncé dans la figure 2 , est maintenant irréversible, en se souvenant toutefois qu'aucun des matériaux à aimant entré dans le cycle industriel n'a disparu des circuits de production et d'utilisation. Quant à leur industrie, elle est des plus dynamiques et est promise à un bel avenir, surtout en cette période où le Monde a pris conscience de la nécessité de sauver de l'énergie. L'aimant moderne est un convertisseur d'énergie, de performance exceptionnelle, car son rendement de transformation est égal à l'unité.

Quelques chiffres suffiront pour le prouver. Il a été calculé [73] que si le nombre d'électrons de notre corps présentait un déficit ou un excès de $1 \%$, nous serions capables de soulever la Terre à bout de bras, par répulsion électrique. Le même calcul, fait pour des masses magnétiques d'origine électronique, donne un nombre égal à $5000 \mathrm{t}$, dans un rapport au premier égal au carré de la vitesse de la lumière $(*)$. C'est peu ; mais c'est aussi beaucoup, car si la première situation n'est pas réalisée par la Nature, qui assure en permanence une neutralisation électrique absolue, la seconde l'est, avec les spins électroniques et orbitaux des éléments de transition à couche interne incomplète. Fabriquer un aimant revient simplement à ordonner parallèlement ces moments magnétiques et à les y maintenir, par des forces que délivre également la Nature, à savoir les couplages spins-orbites. Saturer un aimant consomme de l'énergie, par exemple $10 \mathrm{~kJ}$ dans un banc d'aimantation industriel, pour en récupérer environ $100 \mathrm{~J}$ sous forme d'énergie d'ai-

(*) Ce rapport $1 / c^{2}$ montre combien il sera difficile de maîtriser la répulsion électrique par un champ magnétique pour récupérer de l'énergie en fusion contrôlée. Cela ne sera possible qu'en transformant l'énergie magnétique en énergie électrique par passage aux vitesses relativistes, donc en échauffant suffisamment le plasma. Ceci est réalisé de façon non contrôlée avec une amorce atomique, et le sera peut-être avec l'énergie incidente des lasers. Mais cet horizon est encore lointain, et d'ici là, toutes les sources et économies possibles d'énergie devront être mises à profit. 
mantation; le rendement est apparemment faible, mais il faut se rappeler que $10 \mathrm{~kJ}$ ne sont jamais que l'énergie d'une lampe de $100 \mathrm{~W}$ brûlant inutilement pendant $100 \mathrm{~s}$. Une fois saturé, l'aimant sauve, sa vie durant, de l'énergie gaspillée, en transformant sans pertes la mécanique en électrique ou inversement. A dimensions équivalentes, l'aimant permanent moderne a atteint une telle puissance qu'il dépasse largement, à moindre coût, l'électro-aimant entretenu, sur le plan de l'efficacité volumique.

Quelques chiffres permettront d'estimer le marché potentiel. L'Américain moyen consomme annuellement un aimant de $150 \mathrm{~g}$, l'Européen, un aimant de $100 \mathrm{~g}$, un Habitant du reste du Monde, un aimant de
$10 \mathrm{~g}$. Sans que l'aimant soit un luxe ou un souci de pollution, on appréciera aisément l'énorme capacité de production qu'il faudra mettre en place pour atteindre annuellement les trois millions de tonnes du Futur.

Remerciements. - Au terme de ce tour d'horizon, nous tenons à remercier tous nos collègues et collaborateurs du Laboratoire et de l'Usine, dont la gentillesse et la compétence nous ont permis d'apprendre rapidement en quelques années les notions fondamentales du magnétisme technique et dont les travaux ont été largement mis à contribution pour illustrer cet article.

\section{Bibliographie}

[1] Faure, A., Techniques C. E. M. 88 (1973) 3.

[2] Hubbard, W. M., Adams, E. et Gilfrich, J. V., J. Appl. Phys. 31 (1960) 3685.

[3] Strnat, K. J., Cobalt 36 (1967) 133.

[4] DAS, D. K., I. E. E. E. Magnetics 5 (1969) 214.

[5] Barbara, B., Becle, C. et Lemaire, R., I. E. E. E. Magnetics 7 (1971) 654.

[6] BeCKer, J. J., Met. Rev. 7 (1962) 371.

[7] Shanley, C. W. et Harmer, R. S., 19th Conference on Magnetism and Magnetic Materials, Boston 1973.

[8] JACOBs, I. S., J. Appl. Phys. 40 (1969) 917.

[9] Cullity, B. D., "Introduction to Magnetic Materials » (Addison-Wesley Publishing Cy) 1972.

[10] Kersten, M., Z. Phys. 44 (1943) 63.

[11] NéEL, L., Cahiers Phys. 25 (1944) 19.

[12] Williams, H. J., Bozorth, R. M. et Shockley, W., Phys. Rev. 75 (1949) 155.

[13] McCurrie, R. A. et Gaunt, P., Proc. Intern. Conf. Magnetism, Nottingham 1964, Institute of Physics and the Physical Society, 1965, London, 780.

[14] Marque déposée des aimants UGIMAG S. A., ex. AllevardUgine.

[15] Becker, J. J., Luborsky, F. E. et Martin, D. L., I. E. E. E. Magnetics 4 (1968) 84.

[16] Stoner, E. C. et Wohlfarth, E. P., Phil. Trans. Roy. Soc. London 240A (1948) 599.

[17] Falk, R. B., J. Appl. Phys. 37 (1966) 1108.

[18] Jacobs, I. S. et Bean, C. P., Phys. Rev. 100 (1955) 1060.

[19] BRONNER, C., Résultats non publiés.

[20] Frei, E. H., Shtrikman, S. et Treves, D., Phys. Rev. 106 (1957) 446.

[21] Nesbitt, E. A., Ghin, G. Y., Hull, G. W., Sherwood, R. C., Green, M. L. et Wernick, J. H., A. I. P. Conf. Proc. 10 (1973) 593.

[22] Leamy, H. J. et Green, M. L., I. E. E. E. Magnetics 9 (1973) 205.

[23] Zijlstra, H., J. Appl. Phys. 42 (1971) 1510.

[24] Becker, J. J., J. Appl. Phys. 42 (1971) 1537.

[25] Brown, W. F., Rev. Mod. Phys. 17 (1945) 15.

[26] BeCKer, J. J., I. E. E. E. Magnetics 9 (1973) 161.

[27] AlfF, E., Givord, D. et HABerer, J. P., I. E. E. E. Magnetics 9 (1973) 631.
[28] Mishima, T., Brevet « Alloy for Permanent Magnets », 1932

[29] Olivier, D. A. et Sweden, J. W., Nature 142 (1938) 209.

[30] Bronner, C., «Journée des Aimants Permanents », Paris 1965, Chambre Syndicale des Aciers Spéciaux.

[31] De Vos, K. J., " Alnico Permanent Magnet Alloys », dans " Magnetism and Metallurgy », édité par Berkowitz A. E. et Kneller E. (Academic Press) 1969, Vol. 1.

[32] Iwama, Y. et TaKeuchi, M., Conférence Intermag, Tokyo 1972.

[33] Bronner, C., Haberer, J. P., Planchard, E., Sauze, J., Drapier, J. M., Coutsouradis, D. et Habraken, L., Cobalt 40 (1968) 131 et 42 (1969) 14.

[34] NéEL, L., « Journées des Aimants Permanents », Paris 1969, Chambre Syndicale des Aciers Spéciaux.

[35] Brown, W. F., "Magnetostatic Principles in Ferromagnetism » (North Holland Cy) 1962.

[36] Harrold, W. J., AIP Conference Proceedings 5 (1971) 1006.

[37] BLondot, C., Communication personnelle.

[38] Guillaud, C., Thèse de Doctorat d'Etat, Strasbourg, 1943.

[39] Went, J. J., Rathenau, G. W., Gorter, E. W. et Van Oosterhout, G. W., Philips Tech. Rev. 13 (1952) 194.

[40] Adelsköld, Arkivf. Kemi, Miner. och. Geol. 12-A (1938) 1.

[41] Lemaire, H., «Journée des Aimants Permanents », Paris 1973, Chambre Syndicale des Aciers Spéciaux.

[42] Minnesota Mining and Manufacturing Cy, Brevet français, 1971, $\mathrm{n}^{0} 2104251$.

[43] Gillam, E. et Smethurst, E., Proc. Brit. Cer. Soc. 2 (1964) 129.

[44] Frei, E. H., Shtrikman, S. et Treves, D., Z. Angew. Phys. 21 (1966) 137.

[45] The Rank Organisation Ltd, Brevet français, 1972, No 2137567.

[46] Wernick, J. H. et Geller, S., Acta Crystallogr. 12 (1959) 662.

[47] Becker, J. J., J. Appl. Phys. 41 (1970) 1055.

[48] Wallace, W. E., « Rare Earth Intermetallics 》 (Academic Press, New York) 1973.

[49] Lemaire, R., Cobalt 32 et 33 (1966) 132 et 201.

[50] Ray, A. E., Biermann, A. T., Harmer, R. S. et Davison J. E., Cobalt 4 (1973) 103.

[51] Martin, D. L., Benz, M. G., et Rockwood, A. C., A. I. P. Conf. Proc., 10 (1972) 583. 
[52] Haberer, J. P., Journée des Aimants Permanents 1973, Chambre Syndicale des Aciers Spéciaux, Paris.

[53] Bronner, C., Monographie des Aimants Permanents, 1971, Centre International du Cobalt, Bruxelles.

[54] Den Broeder, F. J. A. et Buschow, K. H. J., J. LessCommon Met. 29 (1972) 65.

[55] Smeggil, J. G., Rao, P., Livingston, J. D. et Koch, E. F., 1973, Conference on Magnetism and Magnetic Materials, Boston.

[56] Mac Currie, R. A., Cobalt 1 (1973) 23.

[57] StÄBleIN, H., IEEE Magnetics 6 (1970) 172.

[58] Dietrich, H., « Ferrites » (University Park Press) 1970, 283.

[59] Tanasoru, C., IEEE Magnetics 8 (1972) 348.

[60] HABerer, J. P. et Lemaire, H., Résultats non publiés.

[61] DuRAND, E., «Magnétostatique » (Masson Editeurs, Paris) 1968.

[62] Iden, D. J., Ehrenfried, C. E. et Garrett, H. J., AIP Conf. Proc. 5 (1971) 1026.

[63] MaC CaIG, M., «Permanent Magnets for Repulsion
Devices 》 (Permanent Magnet Association, Sheffield) 1960.

[64] Polgreen, G. R., « New Applications of Modern Magnets » (Mac Donald, London) 1966.

[65] Cullen, T. J., J. Appl. Phys. 42 (1971) 1535.

[66] Parker, R. J. et Studders, R. J., "Permanent Magnets and their Application » (John Wiley and Sons) 1962, p. 25.

[67] Benz, M. G. et Martin, D. L., J. Appl. Phys. 43 (1972) 4733.

[68] SMEGGIL, J. G., IEEE Magnetics 9 (1973) 158.

[69] Westendorp, F. F., Solid State Commun. 8 (1970) 139.

[70] Bartholin, H., 1966, Thèse de Doctorat, Grenoble.

[71] Nesbitt, E. A., Chin, G. Y., Gallagher, P. K., Sherwood, R. C. et Wernick, J. H., J. Appl. Phys. 42 (1971) 1530.

[72] Benz, M. G., Laforce, R. P. et Martin, D. L., 19th Conference on Magnetism and Magnetic Materials, Boston 1973.

[73] Feynman, R. P., Leighton, R. B. et Sands, M., The Feynman Lectures on Physics (Addison-Wesley Publishing Cy) 1969, Vol. 2, I-1. 